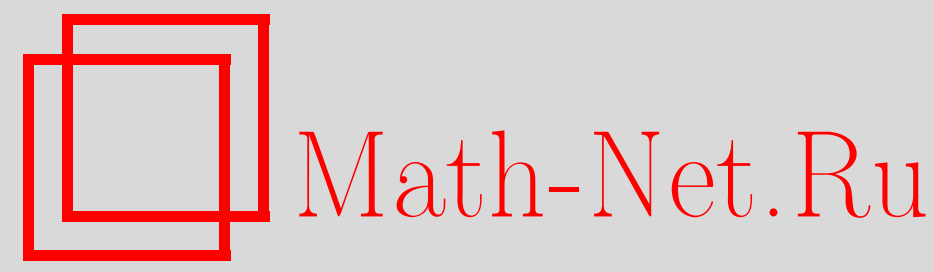

В. В. Щиголев, О некоторых расширениях вполне расщепляемых модулей, Изв. РАН. Сер. матем., 2004, том 68, выпуск 4, 205-224

DOI: https://doi.org/10.4213/im499

Использование Общероссийского математического портала Math-Net.Ru подразумевает, что вы прочитали и согласны с пользовательским соглашением http: //www.mathnet.ru/rus/agreement

Параметры загрузки:

IP: 3.93 .64 .190

26 апреля 2023 г., 14:55:05 
УДК 512.547 .2

\title{
В. В. Шиголев
}

\section{О некоторых расширениях вполне расшепляемых модулей}

\author{
Получена формула для $\operatorname{Ext}_{K \Sigma_{r}}^{1}\left(D^{\lambda}, D^{\mu}\right)$, где $K$ - поле характеристики бо-

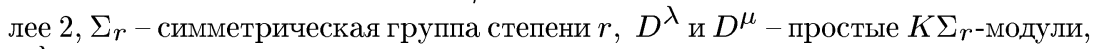 \\ $D^{\lambda}$ - вполне расщепляемый модуль и $\lambda$ не доминирует строго $\mu$. \\ Библиография: 18 наименований.
}

\section{§1. Введение}

В настоящей работе фиксируем некоторое поле $K$ положительной характеристики $p$. Через $\Sigma_{n}$ обозначим симметрическую группу степени $n$ и считаем, что $\Sigma_{m}$ - подгруппа группы $\Sigma_{n}$ при $m<n$. Разбиением натурального числа $n$ назовем последовательность натуральных чисел $\lambda=\left(\lambda_{1}, \ldots, \lambda_{k}\right)$ такую, что $\lambda_{1} \geqslant \cdots \geqslant \lambda_{k} \geqslant 1$ и $\lambda_{1}+\cdots+\lambda_{k}=n$. Последнее обозначим формулой $\lambda \vdash n$. Если при этом $\lambda$ не содержит $p$ и более одинаковых вхождений, то разбиение $\lambda$ называется $p$-регулярным. Далее для любого набора $a$ и натурального числа $i$ через $a_{i}$ будем обозначать $i$-й элемент $a$, если $i$ не превосходит длины $a$, и $a_{i}=0$ в противном случае. Для двух наборов $x=\left(x_{1}, \ldots, x_{n}\right)$ и $y=\left(y_{1}, \ldots, y_{m}\right)$ через $x y$ обозначим набор $\left(x_{1}, \ldots, x_{n}, y_{1}, \ldots, y_{m}\right)$, а в случае, когда эти наборы имеют одинаковую длину и состоят из целых чисел, обозначим через $\alpha x+\beta y$, где $\alpha, \beta \in \mathbb{Z}$, их поэлементную линейную комбинацию. Выражение вида $\left(a_{1}, \ldots, a_{n}\right)$, где $a_{i}-$ либо набор, либо выражение $x^{m}$, будем понимать следуюшим образом. Положим $b_{i}=a_{i}$, если $a_{i}-$ набор, и положим $b_{i}$ равным набору длины $m$, все вхождения которого равны $x$, если $a_{i}$-выражение $x^{m}$. Тогда считаем, что $\left(a_{1}, \ldots, a_{n}\right)=b_{1} \ldots b_{n}$. Например, если $a=(x, y)$ и $b=(z)$, то $\left(a, 1^{3}, b\right)=(x, y, 1,1,1, z)$.

Пусть $\lambda$ и $\mu$-наборы целых чисел. Если для любого $m \in \mathbb{N}$ вьполнено неравенство

$$
\sum_{i=1}^{m} \lambda_{i} \geqslant \sum_{i=1}^{m} \mu_{i}
$$

то считается, что $\lambda$ доминирует $\mu$. Это обозначается $\lambda \unrhd \mu$. Если $\lambda \neq \mu$, то считается, что $\lambda$ строго доминирует $\mu$, и используется обозначение $\lambda \triangleright \mu$. Полученное отношение $\unrhd$ является отношением частичного порядка на множестве разбиений произвольного числа.

Пусть $M, A$ и $B$ - произвольные $K \Sigma_{r}$-модули. Обозначим через $\operatorname{rad} M$ пересечение всех максимальных $K \Sigma_{r}$-подмодулей модуля $M$ (определение $\operatorname{Ext}_{K \Sigma_{r}}^{1}(A, B)$ см., например, в [2, предложение 3 из $\S 5.5]$; там опущен верхний индекс).

Каждому разбиению $\lambda$ числа $n$ соответствует $K \Sigma_{n}$-модуль $S^{\lambda}$, который называется модулем Шnехта (см., например, [1, определение 4.3]). Положим 
$D^{\lambda}=S^{\lambda} / \operatorname{rad} S^{\lambda}$. Отображение $\lambda \mapsto D^{\lambda}$ задает взаимно однозначное соответствие между $p$-регулярными разбиениями числа $n$ и простыми $K \Sigma_{n}$-модулями. Для любого разбиения $\lambda$ через $h(\lambda)$ обозначим длину набора $\lambda$ и назовем это число высотой разбиения $\lambda$. Также положим $\chi(\lambda)=\lambda_{1}-\lambda_{h(\lambda)}+h(\lambda)$.

С произвольным разбиением $\mu$ числа $n$ можно связать подгруппу Юнга $\Sigma_{\mu}$ группы $\Sigma_{n}$ следуюшим способом. Рассмотрим произвольное разбиение $\{1, \ldots, n\}=$ $A_{1} \sqcup \cdots \sqcup A_{h(\mu)}$ такое, что $\left|A_{i}\right|=\mu_{i}$. Тогда положим

$$
\Sigma_{\mu}=\left\{\sigma \in \Sigma_{n}: \sigma A_{i}=A_{i}, i=1, \ldots, h(\mu)\right\} .
$$

Такое определение зависит от выбора множеств $A_{i}$. Однако все подгруппы, получающиеся при различных допустимых $A_{i}$, сопряжены. Поэтому в следуюшем определении не важно, какое именно разбиение рассматривается.

ОПРЕДЕЛЕНИЕ $[16$, п. 0.1$]$. Неприводимый $K \Sigma_{n}$-модуль $D^{\lambda}$ называется вполне расщепляемым тогда и только тогда, когда ограничение $D^{\lambda} \downarrow_{\Sigma_{\mu}}$ на любую подгруппу Юнга $\Sigma_{\mu} \subset \Sigma_{n}$ полупросто.

ТЕОРема [16, п. 2.1]. Модуль $D^{\lambda}$ вполне расщепляем тогда и только тогда, когда $\chi(\lambda) \leqslant p$.

В работе [8] была получена формула для $\operatorname{Ext}_{\Sigma_{n}}^{1}\left(D^{\lambda}, D^{\mu}\right)$ в случае, когда $\operatorname{char} K>2$ и оба модуля $D^{\lambda}$ и $D^{\mu}$ вполне расщепляемы. В силу симметрии достаточно ограничиться рассмотрением случая $\lambda \not \mu$. С другой стороны, в работе [3] было доказано, что $\operatorname{Ext}_{\Sigma_{n}}^{1}\left(D^{\lambda}, D^{\mu}\right)=0$, если $D^{\lambda}$ - вполне расщепляемый модуль, $h(\mu) \leqslant h=h(\lambda)$ и $\lambda_{h}-\mu_{h} \geqslant(h-1)(p-h)+2$ (здесь полагаем $\mu_{h}=0$, если $h(\mu)<h)$.

Основная цель настоящей работы - получить формулу для $\operatorname{Ext}_{\Sigma_{n}}^{1}\left(D^{\lambda}, D^{\mu}\right)$ в случае, когда char $K>2, D^{\lambda}$ - вполне расщепляемый модуль и $\lambda \not \mu$ (см. теорему 6), и, таким образом, усилить два упомянутых вьше результата. Заметим, что в работе [13] вычислены пространства $\operatorname{Ext}_{\Sigma_{n}}^{1}\left(D^{\lambda}, D^{\mu}\right)$ в случае, когда char $K>2$ и $h(\lambda), h(\mu) \leqslant 2$. Проблеме расширений неприводимых $K \Sigma_{r}$-модулей посвящена также работа [12], где изучается связь таких расширений с расширениями модулей над алгеброй Шура и обшей линейной группой.

Для разбиения $\lambda$ числа $n$ определим его диаграмму Юнга формулой $[\lambda]=$ $\left\{(i, j) \in \mathbb{N} \times \mathbb{N}: i \leqslant h(\lambda), j \leqslant \lambda_{i}\right\}$. Элементы из $\mathbb{N} \times \mathbb{N}$ будем называть клетками. Положим $r(i, j)=i$ и $c(i, j)=j$. Предполагается, что клетка $A$ расположена выше клетки $B$, если $r(A)<r(B)$, и левее клетки $B$, если $c(A)<c(B)$. Клетка $C$ считается расположенной между клетками $A$ и $B$, если $r(A)<r(C)<r(B)$ или $r(B)<r(C)<r(A)$. Класс вычетов $(j-i)+p \mathbb{Z}$ называется вычетом клетки $(i, j)$ и обозначается $\operatorname{res}(i, j)$. Если диаграммы разбиений $\lambda$ и $\mu$ содержат одинаковое количество клеток каждого вычета, то будем записьвать $\lambda \sim \mu$. Если $A=\left(i, \lambda_{i}\right)$, где $i<h(\lambda)$ и $\lambda_{i}>\lambda_{i+1}$ или $i=h(\lambda)$, то клетка $A$ называется $\lambda$-yдаляемой и через $\lambda_{A}$ обозначается разбиение, диаграмма которого равна $[\lambda] \backslash\{A\}$ (конечно, если $[\lambda]$ содержит более одной клетки). Если $B=\left(i, \lambda_{i}+1\right)$ и $i \leqslant h(\lambda)$ или $B=(h(\lambda)+1,1)$, то клетка $B$ называется $\lambda$-добавляемой и через $\lambda^{B}$ обозначается разбиение, диаграмма которого равна $[\lambda] \cup\{B\}$. Обозначим также через $\lambda^{t}$ разбиение, диаграмма которого получена транспонированием диаграммы $[\lambda]$. 
В работе [4] были введены следуюшие определения (хотя некоторые из них были сформулированы ранее). Клетка $A$ называется $\lambda$-нормальной, если эта клетка $\lambda$-удаляемая и для любой $\lambda$-добавляемой клетки $B$ вычета $\operatorname{res} A$, расположенной выше $A$, сушествует $\lambda$-удаляемая клетка $C(B)$ того же вычета между $A$ и $B$, и при этом из $B \neq B^{\prime}$ следует $C(B) \neq C\left(B^{\prime}\right)$. Самая нижняя из $\lambda$-нормальных клеток данного вычета называется $\lambda$-хорошей. Клетка $A$ называется $\lambda$-конормальной, если эта клетка $\lambda$-добавляемая и для любой $\lambda$-удаляемой клетки $B$ вычета res $A$, расположенной ниже $A$, существует $\lambda$-добавляемая клетка $C^{\prime}(B)$ того же вычета между $A$ и $B$, и при этом из $B \neq B^{\prime}$ следует $C^{\prime}(B) \neq C^{\prime}\left(B^{\prime}\right)$. Самая верхняя из $\lambda$-конормальных клеток данного вычета называется $\lambda$-кохорошей.

Имеют место следующие утверждения, доказанные в [13, теорема 2.10], [14], $[15]$ и $[4]$.

УТВЕРЖДЕНИЕ 1. Пусть $p>2 u \lambda, \mu$-разбиения числа $n$, имеющие не более $p-1$ (ненулевой) части. Если $\lambda \ngtr \mu$, mо $\operatorname{Ext}_{\Sigma_{n}}^{1}\left(D^{\lambda}, D^{\mu}\right) \cong \operatorname{Hom}_{\Sigma_{n}}\left(\operatorname{rad} S^{\lambda}, D^{\mu}\right)$.

УТВЕРЖДЕНИЕ 2. Пусть имеются р-регулярные разбиения $\lambda \vdash r u \mu \vdash r+1$. Тогда:

1) $\operatorname{Hom}_{\Sigma_{r}}\left(S^{\lambda}, D^{\mu} \downarrow_{\Sigma_{r}}\right)$ одномерно, если $\lambda=\mu_{A}$ для некоторой $\mu$-нормальной клетки $A$, иначе равно нулю;

2) $\operatorname{Hom}_{\Sigma_{r}}\left(D^{\lambda}, D^{\mu} \downarrow_{\Sigma_{r}}\right)$ одномерно, если $\lambda=\mu_{A}$ для некоторой $\mu$-хорошей клетки $A$, иначе равно нулю;

3) $\operatorname{Hom}_{\Sigma_{r+1}}\left(S^{\mu}, D^{\lambda} \uparrow^{\Sigma_{r+1}}\right)$ одномерно, если $\mu=\lambda^{B}$ для некоторой $\lambda$-конормальной клетки $B$, иначе равно нулю;

4) $\operatorname{Hom}_{\Sigma_{r+1}}\left(D^{\mu}, D^{\lambda} \uparrow \Sigma_{r+1}\right)$ одномерно, если $\mu=\lambda^{B}$ для некоторой $\lambda$-кохорошей клетки $B$, иначе равно нулю.

В $\S 2,3$ мы установим справедливость некоторых неравенств, включающих размерности пространств $\operatorname{Ext}_{\Sigma_{r}}^{1}\left(D^{\lambda}, D^{\mu}\right)$ и $\operatorname{Ext}_{\Sigma_{r}}^{\mathrm{e}}\left(D^{\lambda}, D^{\mu}\right)$, где $\lambda$ и $\mu$ - разбиения высоты не более $p$. В $\S 2$ проведем подготовку того, чтобы сделать возможным рассмотрение случая разбиений высоты $p$. Хотя в этом случае эквивалентность утверждения 1 не всегда верна, мы вводим “функтор" Ext ${ }^{\mathrm{e}}$, в силу теоремы 1 связанный с пространством Ноm $\Sigma_{r}\left(\operatorname{rad} S^{\lambda}, D^{\mu}\right)$. Изучение этого пространства важно для изучения поднятия радикала модулей Шпехта $S^{\lambda}$, описанного в [18], которое можно рассматривать как операцию приписывания столбца высоты не менее $h(\lambda)$. Читатель, желающий ограничиться рассмотрением случая разбиений высоты менее $p$, может пропустить $\S 2$ и заменить везде в тексте введенный в этом параграфе функтор $\mathrm{Ext}^{\mathrm{e}}$ на $\mathrm{Ext}^{1}$. Следует заметить, что если $D^{\lambda}$ - вполне расшепляемый мо-

дуль, то $h(\lambda)<p$, так как $\chi(\lambda) \leqslant p$ и $\lambda-p$-регулярное разбиение. Наконец, именно при помощи неравенств из $\S 3$ в $\S 4$ получен основной результат работы.

Автор благодарит А. С. Клещева за полезную консультацию.

\section{§ 2. Продолжаемые модули}

В этом параграфе будем использовать теорию функтора Шура, описанную в $[7, \S 6.1]$. Обозначим через $I(n, r)$ множество последовательностей длины $r$, состоящих из натуральных чисел от 1 до $n$. Для любых $i \in I(n, r), \pi \in \Sigma_{n}$ и $\sigma \in \Sigma_{r}$ положим $\pi i=\left(\pi\left(i_{1}\right), \ldots, \pi\left(i_{r}\right)\right)$ и $i \sigma=\left(i_{\sigma(1)}, \ldots, i_{\sigma(r)}\right)$. Заметим, что тогда $(\pi i) \sigma=\pi(i \sigma)$. 
Через $S(n, r)$ обозначим алгебру Шура с $K$-базисом $\xi_{i, j}$, где $i, j \in I(n, r)$. (Определения см. в $[7, \S 2.3]$; мы опустили индекс в обозначении алгебры Шура.) Для любого натурального числа $r$ фиксируем набор $u(r)=(1, \ldots, r)$ и идемпотент $e_{r}=\xi_{u(r), u(r)}$. Для $m \geqslant r$ определим вложение $K$-алгебр $K \Sigma_{r} \rightarrow S(m, r)$ по формуле $\sigma \mapsto \xi_{u(r) \sigma, u(r)}$. Это вложение задает изоморфизм алгебр $K \Sigma_{r}$ и $e_{r} S(m, r) e_{r}$. Поэтому мы будем рассматривать любой $e_{r} S(m, r) e_{r}$-модуль как $K \Sigma_{r}$-модуль, и наоборот.

Обозначим через $\Lambda(n, r)$ множество таких последовательностей $\left(\lambda_{1}, \ldots, \lambda_{n}\right)$, что $\lambda_{1}, \ldots, \lambda_{n}$ - неотрицательные целые числа и $\lambda_{1}+\cdots+\lambda_{n}=r$. Подмножество множества $\Lambda(n, r)$, состоящее из последовательностей $\lambda$ таких, что $\lambda_{1} \geqslant \cdots$ $\cdots \geqslant \lambda_{n}$, обозначим через $\Lambda^{+}(n, r)$. Набор $\alpha \in \Lambda^{+}(n, r)$ называется $p$-регулярныцм по столбцам, если $\alpha_{i}-\alpha_{i+1}<p$ для любого $i=1, \ldots, n-1$ и $\alpha_{n}<p$.

Через $\operatorname{sgn}_{r}$ обозначим знакопеременное представление групाы $\Sigma_{r}$. С этим представлением связан еше один способ нумеровать представления: положим $D_{\lambda^{t}} \cong$ $D^{\lambda} \otimes \operatorname{sgn}_{r}$, где $\lambda$ - любое $p$-регулярное разбиение. Для натурального числа $m$ и разбиения $\lambda$ высоты не более $m$ положим $(\lambda)_{m}=\left(\lambda, 0^{m-h(\lambda)}\right)$. Через $\Delta(\lambda)$ и $L(\lambda)$, где $\lambda \in \Lambda^{+}(n, r)$, мы обозначим соответственно модуль Вейля и неприводимый модуль со старшим весом $\lambda$. В работе [7] эти модули обозначались через $V_{\lambda, K}$ и $F_{\lambda, K}$ (определения см. в $[7, \S 5.2$ и теорема $3.5 \mathrm{a}]$ ).

ОПРЕДЕЛЕНИЕ 1 . Пусть $M$ - некоторый $K \Sigma_{r}$-модуль и $\lambda$ - разбиение числа $r$. Модуль $M$ назовем $\lambda$-продолжаемым, если сушествует такой $S(r, r)$-модуль $N$, что вьполнены условия:

1) если $\mu$ - вес модуля $N$, то $\mu \unlhd\left(\lambda^{t}\right)_{r}$;

2) $M \otimes \operatorname{sgn}_{r} \cong e_{r} N$.

В этом случае модуль $N$ называется $\lambda$-продолжением модуля $M$.

ЗАмЕчАНИЕ 1. Из этого определения непосредственно следует, что если $\lambda^{\prime} \unlhd \lambda$ и $M-\lambda$-продолжаемый модуль, то $M-\lambda^{\prime}$-продолжаемый модуль.

ЛЕмма 1. Класс $\lambda$-продолжаемых модулей замкнут относительно перехода к подмодулям, фактормодулям, двойственным модулям и образования конечных пряммых сумм.

ДОКАЗАТЕЛЬСТВО. Пусть $M$ - некоторый $\lambda$-продолжаемый $K \Sigma_{r}$-модуль, $L-$ его подмодуль и $M^{*}$ - модуль, двойственный модулю $M$. Пусть $N-\lambda$-продолжение модуля $M$ и $f: M \otimes \operatorname{sgn}_{r} \rightarrow e_{r} N-$ изоморфизм. Положим $T=S(r, r) f\left(L \otimes \operatorname{sgn}_{r}\right)$. При этом $e_{r} T=f\left(L \otimes \operatorname{sgn}_{r}\right)$. Определим $K$-линейные отображения

$$
f^{\prime}: L \otimes \operatorname{sgn}_{r} \rightarrow e_{r} T \quad \text { и } \quad f^{\prime \prime}:(M / L) \otimes \operatorname{sgn}_{r} \rightarrow e_{r}(N / T)
$$

следуюшим образом:

$$
\begin{gathered}
f^{\prime}(x)=f(x), \quad x \in L \otimes \operatorname{sgn}_{r}, \\
f^{\prime \prime}((y+L) \otimes v)=f(y \otimes v)+T, \quad y \in M, \quad v \in \operatorname{sgn}_{r} .
\end{gathered}
$$

Последнее отображение задано корректно, так как если $y \in L$, то $f(y \otimes v) \in T$.

Далее, $\operatorname{Ker} f^{\prime}=0$ и

$$
\operatorname{dim} e_{r} T=\operatorname{dim} f\left(L \otimes \operatorname{sgn}_{r}\right)=\operatorname{dim} L \otimes \operatorname{sgn}_{r},
$$


поэтому $f^{\prime}$ изоморфно отображает $L \otimes \operatorname{sgn}_{r}$ на $e_{r} T$. Предположим, что

$$
f^{\prime \prime}((y+L) \otimes v)=0, \quad \text { где } y \in M \text { и } v \in \operatorname{sgn}_{r} \backslash\{0\} .
$$

Тогда

$$
f(y \otimes v) \in e_{r} T=f\left(L \otimes \operatorname{sgn}_{r}\right) .
$$

Отсюда $y \otimes v \in L \otimes \operatorname{sgn}_{r}$ и $y \in L$. С другой стороны,

$$
\begin{aligned}
\operatorname{dim} e_{r}(N / T) & =\operatorname{dim} e_{r} N-\operatorname{dim} e_{r} T \\
& =\operatorname{dim} f\left(M \otimes \operatorname{sgn}_{r}\right)-\operatorname{dim} f\left(L \otimes \operatorname{sgn}_{r}\right)=\operatorname{dim}(M / L) \otimes \operatorname{sgn}_{r}
\end{aligned}
$$

поэтому $f^{\prime \prime}$ изоморфно отображает $(M / L) \otimes \operatorname{sgn}_{r}$ на $e_{r}(N / T)$. Условия 1$)$ и 2$)$ определения 1 теперь проверяются автоматически.

Для доказательства того, что $M^{*}-\lambda$-продолжаемый модуль, достаточно заметить, что $e_{r} N^{\prime} \cong M^{*}$, где $N^{\prime}$ - модуль, контравариантно дуальный модулю $N$. Выполнение условия 1 ) следует из того, что характеры модулей $N$ и $N^{\prime}$ совпадают.

Пусть теперь $M^{\prime}$ и $M^{\prime \prime}-\lambda$-продолжаемые $K \Sigma_{r}$-модули, и пусть $N^{\prime}$ и $N^{\prime \prime}-$ $\lambda$-продолжения модулей $M^{\prime}$ и $M^{\prime \prime}$ соответственно. Легко видеть, что тогда $N^{\prime} \oplus N^{\prime \prime}-\lambda$-продолжение модуля $M^{\prime} \oplus M^{\prime \prime}$.

Установим, какие модули $\lambda$-продолжаемы. Во-первых, очевидно, что любой неприводимый модуль $D^{\lambda}$ является $\lambda$-продолжаемым. Во-вторых, любой модуль $M^{\lambda}$ (см. $[1, \S 4.1])$ также $\lambda$-продолжаем. В качестве его $\lambda$-продолжения можно, например, взять модуль $E^{\otimes r}\{C(T)\}$, где $E \cong K^{r}$ и $T$ - некоторая основная $\lambda^{t}$-таблица (см. $[7, \S 4.2,5.3])$.

Пусть имеется некоторое разбиение $\lambda \vdash r, A_{1}, \ldots, A_{k}$-все $\lambda$-удаляемыеклетки, $B_{1}, \ldots, B_{l}$ - все $\lambda$-добавляемые клетки, перечисленные сверху вниз. Положим $\lambda^{-}=\lambda_{A_{1}}$ и $\lambda^{+}=\lambda^{B_{l-1}}$.

Лемма 2. Пусть $\lambda-$ разбиение числа $r>1$. Тогда $S^{\lambda} \downarrow_{\Sigma_{r-1}}-\lambda^{-}-$продолжаемый модуль.

ДоказАТЕЛЬСтво. В $[1, \S 9.3]$ фактически доказано, что $S^{\mu} \cap \bigcap_{i=1}^{m} \operatorname{Ker} \theta_{i}=0$. Возврашаясь к введенным обозначениям, мы получаем, что сушествует вложение $S^{\lambda} \downarrow_{\Sigma_{r-1}}$ в $\bigoplus_{s=1}^{k} M^{\lambda_{A s}}$, где $A_{1}, \ldots, A_{k}$ - все $\lambda$-удаляемые клетки, перечисленные сверху вниз. Теперь лемма следует из леммы 1 , замечания 1 и того, что $\lambda^{-}=$ $\lambda_{A_{1}} \triangleleft \lambda_{A_{s}}$ для $s=2, \ldots, k$.

ЛЕмма 3. Пусть $\lambda$ - разбиение числа $r$. Тогда существует подмодуль $U$ модуля $S^{\lambda \uparrow \Sigma_{r+1}}$, изоморфный $S^{(\lambda, 1)}$, такой, что $S^{\lambda \uparrow \Sigma_{r+1}} / U-\lambda^{+}{ }_{-}$роодолжаемый модуль.

ДоКАЗАТЕЛЬСТВО. Из $[1, \S 17.13]$ следует, что модуль $S^{\lambda} \uparrow \Sigma_{r+1}$ имеет подмодуль $U$, изоморфный $S^{(\lambda, 1)}$, такой, что $S^{\lambda} \uparrow^{\Sigma_{r+1}} / U$ вложим в $M^{\lambda^{+}}$. Далее применяем лемму 1. 
ОПРЕДЕЛЕНИЕ $2 . K \Sigma_{r}$-модуль $M$ называется продолжсаемы.м, если он $\lambda$-продолжаем для некоторого разбиения $\lambda \vdash r$ высоты не более $p$. Под продолжением модуля $M$ будем понимать любое его $\lambda$-продолжение, где $\lambda$ - разбиение $r$ высоты не более $p$.

Очевидно, что $M$ продолжаем тогда и только тогда, когда он $\left((q+1)^{k}, q^{p-k}\right)$ продолжаем, где $r=p q+k, q, k \in \mathbb{Z}$ и $0 \leqslant k \leqslant p-1$.

ОПРЕДЕЛЕНИЕ 3 . Пусть $\lambda, \mu-p$-регулярные разбиения числа $r$. Если существует продолжаемый $K \Sigma_{r}$-модуль $M$, для которого $\operatorname{soc} M \cong D^{\mu}$ и $M / \operatorname{soc} M \cong$ $\bigoplus m D^{\lambda}$, то обозначим через $n$ максимальное из таких $m$. В противном случае определим $n=0$. Положим $\operatorname{Ext}_{\Sigma_{r}}^{\mathrm{e}}\left(D^{\lambda}, D^{\mu}\right)=K^{n}$.

Ясно, что $\operatorname{dim} \operatorname{Ext}_{\Sigma_{r}}^{\mathrm{e}}\left(D^{\lambda}, D^{\mu}\right) \leqslant \operatorname{dim} \operatorname{Ext}_{\Sigma_{r}}^{1}\left(D^{\lambda}, D^{\mu}\right)$ и что $\operatorname{Ext}_{\Sigma_{r}}^{\mathrm{e}}\left(D^{\lambda}, D^{\mu}\right)=0$, если $h(\lambda)>p$ или $h(\mu)>p$.

ЛЕмма 4. Если $\lambda и \mu-p$-регулярные разбиения числа $r$, mо $\operatorname{Ext}_{\Sigma_{r}}^{\mathrm{e}}\left(D^{\lambda}, D^{\mu}\right) \cong$ $\operatorname{Ext}_{\Sigma_{r}}^{\mathrm{e}}\left(D^{\mu}, D^{\lambda}\right)$.

ДокаЗАТЕльСтво. Достаточно считать, что $h(\lambda), h(\mu) \leqslant p$. Положим $n=$ $\operatorname{dim} \operatorname{Ext}_{\Sigma_{r}}^{\mathrm{e}}\left(D^{\lambda}, D^{\mu}\right)$, и пусть $M-$ продолжаемьй $K \Sigma_{r}$-модуль, для которого $\operatorname{soc} M \cong D^{\mu}$ и $M / \operatorname{soc} M \cong \bigoplus n D^{\lambda}$. Пусть $I-$ индуктивная оболочка модуля $D^{\lambda}$ и $\varphi_{i}: \operatorname{rad} M^{*} \rightarrow I, i=1, \ldots, n,-$ линейно независимые гомоморфизмы, где $M^{*}-$ модуль, двойственный модулю $M$. Обозначим через $\bar{\varphi}_{i}$ продолжение $\varphi_{i}$ до гомоморфизма из $M^{*}$ в $I$. Зададим гомоморфизмы $\psi_{i}: M^{*} / \operatorname{rad} M^{*} \rightarrow I / D^{\lambda}$ формулой

$$
\psi_{i}\left(x+\operatorname{rad} M^{*}\right)=\bar{\varphi}_{i}(x)+D^{\lambda}
$$

Легко видеть, что $\psi_{1}, \ldots, \psi_{n}$ линейно независимы. Теперь если положить $N=$ $\sum_{i=1}^{n} \bar{\varphi}_{i}\left(M^{*}\right)$, то по лемме 1 модуль $N$ продолжаемьй, $\operatorname{soc} N \cong D^{\lambda}$ и $N / \operatorname{soc} N \cong$ $\bigoplus n D^{\mu}$. Следовательно, $n \leqslant \operatorname{dim} \operatorname{Ext}_{\Sigma_{r}}^{\mathrm{e}}\left(D^{\mu}, D^{\lambda}\right)$. Обратное неравенство доказывается аналогично.

Лемма 5. Пусть $\alpha, \beta \in \Lambda^{+}(m, r), m \geqslant r u k<m$-некоторое число такое, что $\left(\alpha_{1}, \ldots, \alpha_{k}\right) \nsupseteq\left(\beta_{1}, \ldots, \beta_{k}\right), \alpha_{k+1}>0$. Предположим, что существует некоторый $\nu \in \Lambda(m, r)$ такой, что:

1) $\nu$ - вес модуля $L(\beta)$;

2) $\nu-\gamma$ не вес модуля $L\left(\alpha_{k+1}, \ldots, \alpha_{m}, 0^{k}\right)$ для любого $\gamma \in \Lambda\left(m, \alpha_{1}+\cdots\right.$ $\left.\cdots+\alpha_{k}\right)$.

Tогда $\operatorname{Ext}_{S(m, r)}^{1}(L(\alpha), L(\beta))=0$.

ДокАЗАТЕЛЬСТвО. Пусть $N$ - произвольный $S(m, r)$-модуль, для которого сушествует подмодуль $N_{0} \subset N$ такой, что $N_{0} \cong L(\beta)$ и $N / N_{0} \cong L(\alpha)$. Пусть $v-$ элемент из $N^{\alpha}$ и $U=S(m, r) v$. Достаточно доказать, что $U \cap N^{\nu}=0$, так как в этом случае $N_{0}$ не может быть подмодулем модуля $U$. Пусть $j \in I(m, r)$ - набор веса $\nu$. Рассмотрим представление $j=j^{(1)} \ldots j^{(m)}$, где $j^{(s)}$ - набор длины $\alpha_{s}$. Положим также $i=\left(1^{\alpha_{1}}, \ldots, m^{\alpha_{m}}\right)$. Имеем

$$
\left|j^{(k+1)} \ldots j^{(m)}\right|=r-\left(\alpha_{1}+\cdots+\alpha_{k}\right) \leqslant r-k \leqslant m-k .
$$


Поэтому существует некоторая перестановка $\sigma \in \Sigma_{m}$ такая, что любая последовательность $\sigma j^{(s)}, s=k+1, \ldots, m$, не содержит чисел $1, \ldots, k$. Положим

$$
\tilde{j}^{(s)}=\sigma j^{(s)}, \quad l=\left(1^{\alpha_{1}}, \ldots, k^{\alpha_{k}}\right) \tilde{j}^{(k+1)} \ldots \tilde{j}^{(m)} .
$$

Имеет место представление $\xi_{j, i}=\xi_{j, l} \xi_{l, i}$ (см., например, [7, $\left.2.3 \mathrm{~b}\right]$ ). Пусть $\gamma \in \Lambda\left(m, \alpha_{1}+\cdots+\alpha_{k}\right)$ - вес последовательности $j^{(1)} \ldots j^{(k)}$. Тогда $\nu-\gamma-$ вес последовательности $j^{(k+1)} \ldots j^{(m)}$, а $(\nu-\gamma) \sigma^{-1}-$ вес последовательности $\tilde{j}^{(k+1)} \ldots \tilde{j}^{(m)}$. Запишем этот вес в виде $(\nu-\gamma) \sigma^{-1}=\left(\varepsilon_{1}, \ldots, \varepsilon_{m}\right)$. При этом $\varepsilon_{1}=\cdots=\varepsilon_{k}=0$.

Используя стандартный метод (см., например, $[10, \S 2])$ удаления первых $k$ строк, получим

$$
\begin{aligned}
L(\alpha)^{\left(\alpha_{1}, \ldots, \alpha_{k}, \varepsilon_{k+1}, \ldots, \varepsilon_{m}\right)} & \cong L\left(\alpha_{k+1}, \ldots, \alpha_{m}\right)^{\left(\varepsilon_{k+1}, \ldots, \varepsilon_{m}\right)} \\
& \cong L\left(\alpha_{k+1}, \ldots, \alpha_{m}, 0^{k}\right)^{\nu-\gamma}=0 .
\end{aligned}
$$

Поскольку $\left(\alpha_{1}, \ldots, \alpha_{k}, \varepsilon_{k+1}, \ldots, \varepsilon_{m}\right)$ - вес последовательности $l$, то получаем $\xi_{l, i} v \in N_{0}$. Однако если $\xi_{l, i} v \neq 0$, то $\left(\alpha_{1}, \ldots, \alpha_{k}, \varepsilon_{k+1}, \ldots, \varepsilon_{m}\right)$ - вес элемента $\xi_{l, i} v$. Так как $\left(\alpha_{1}, \ldots, \alpha_{k}, \varepsilon_{k+1}, \ldots, \varepsilon_{m}\right) \not \beta$ и $\beta$ - старший вес модуля $N_{0} \cong L(\beta)$, то в этом случае получаем противоречие. Отсюда $\xi_{l, i} v=0$ и $U \cap N^{\nu}=0$ в силу произвольности выбора набора $j \in I(m, r)$ веса $\nu$.

ЗАмечАниЕ 2 . В лемме 5 достаточно потребовать $r \leqslant m-k+\alpha_{1}+\cdots+\alpha_{k}$.

СлЕДСТВИЕ 1. Пусть $\beta \in \Lambda^{+}(m, r)-p$-регулярный по столбиам вес, $m \geqslant r$, $r$ делится нар и $\left(p^{(r / p)-1}\right) \not\left(\beta_{1}, \ldots, \beta_{(r / p)-1}\right)$. Тогда

$$
\operatorname{Ext}_{S(m, r)}^{1}\left(L\left(p^{r / p}, 0^{m-(r / p)}\right), L(\beta)\right)=0 .
$$

ДоКАЗАТЕЛЬСТВО. Достаточно применить предыдущую лемму для случая $\alpha=$ $\left(p^{r / p}, 0^{m-(r / p)}\right), k=(r / p)-1$ и $\nu=\left(1^{r}, 0^{m-r}\right)$.

Лемма 6. Пусть $\beta \in \Lambda^{+}(m, p), \quad k \geqslant 1, \quad m \geqslant p u \beta_{1} \leqslant p-2$. Тогда $\operatorname{Ext}_{S(k-1+m, p k)}^{1}\left(L\left(p^{k}, 0^{m-1}\right), L\left(p^{k-1}, \beta\right)\right)=0$.

ДоКАЗАТЕЛЬСТво. Применим тот же прием, что и в лемме 5 . Пусть $N-$ произвольный $S(k-1+m, p k)$-модуль, для которого существует подмодуль $N_{0} \subset N$ такой, что $N_{0} \cong L\left(p^{k-1}, \beta\right)$ и $N / N_{0} \cong L\left(p^{k}, 0^{m-1}\right)$. Положим $\nu=\left(p^{k-1}, 1^{p}, 0^{m-p}\right)$. Используя стандартный метод удаления первых $k-1$ строк и то, что $\beta$ - $p$-регулярный по столбцам вес, получаем

$$
L\left(p^{k-1}, \beta\right)^{\nu} \cong L(\beta)^{\left(1^{p}, 0^{m-p}\right)} \neq 0 .
$$

Пусть $v \in N^{\left(p^{k}, 0^{m-1}\right)}$ и $U=S(k-1+m, p k) v$. Достаточно доказать, что $U \cap N^{\nu}=0$. Пусть $j \in I(k-1+m, p k)$ - набор веса $\nu$ и $j=j^{(1)} \ldots j^{(k)}$, где $j^{(1)}, \ldots, j^{(k)}$ - наборы длины $p$. Положим также $i=\left(1^{p}, \ldots, k^{p}\right)$. Поскольку $j$ имеет вес $\nu$, то существует некоторое $t=1, \ldots, k$ такое, что $j^{(t)}$ содержит по крайней мере два различных числа. Положим

$$
l=\left(1^{p}, \ldots,(t-1)^{p}, t^{p-1}, k+1,(t+1)^{p}, \ldots, k^{p}\right) .
$$

Так как $k-1+m \geqslant k+1$, то $l \in I(k-1+m, p k)$. 
Имеем $\xi_{j, i}=\frac{1}{x} \xi_{j, l} \xi_{l, i}$, где $x-$ количество вхождений числа $j_{p}^{(t)}$ в набор $j^{(t)}$. В силу выбора $t$ мы имеем $1 \leqslant x \leqslant p-1$, и деление корректно. Если $\xi_{l, i} v \neq 0$, то $\left(p^{t-1}, p-1, p^{k-t}, 1,0^{m-2}\right)-$ вес элемента $\xi_{l, i} v$. Имеем

$$
\begin{aligned}
& L\left(p^{k}, 0^{m-1}\right)^{\left(p^{t-1}, p-1, p^{k-t}, 1,0^{m-2}\right)} \cong L\left(p^{k}, 0^{m-1}\right)^{\left(p^{k-1}, p-1,1,0^{m-2}\right)} \\
& \cong L\left(p, 0^{m-1}\right)^{\left(p-1,1,0^{m-2}\right)}=0 \\
& L\left(p^{k-1}, \beta\right)^{\left(p^{t-1}, p-1, p^{k-t}, 1,0^{m-2}\right)} \cong L\left(p^{k-1}, \beta\right)^{\left(p^{k-1}, p-1,1,0^{m-2}\right)} \\
& \cong L(\beta)^{\left(p-1,1,0^{m-2}\right)}=0 .
\end{aligned}
$$

Последнее равенство следует из $\beta_{1} \leqslant p-2$. Теперь, как и в лемме 5 , получаем сначала $\xi_{l, i} v \in N_{0}$, а затем $\xi_{l, i} v=0$. Приходим к противоречию. В силу произвольности выбора набора $j \in I(k-1+m, p k)$ веса $\nu$ получаем $U \cap N^{\nu}=0$.

Следующее утверждение вытекает из $[11, \mathrm{II} .2 .14]$ и $[5$, п. 2.1f].

УТВЕРЖДЕНИЕ 3. Пусть $\alpha, \beta \in \Lambda^{+}(m, r) u \beta \not \alpha$. Тогда

$$
\operatorname{Ext}_{S(m, r)}^{1}(L(\alpha), L(\beta)) \cong \operatorname{Hom}_{S(m, r)}(\operatorname{rad} \Delta(\alpha), L(\beta))
$$

Докажем следующий аналог утверждения 1.

ТЕОРема 1. Пусть $\lambda u \mu-p$-регулярные разбиения числа $r$ такие, что $h(\lambda) \leqslant p, \lambda \not \mu$ в случае, когда $r$ делится на $p$, хотя бы одно из разбиений $\lambda$ или н отлично от $\left((r / p)+1,(r / p)^{p-2},(r / p)-1\right)$. Тогда

$$
\operatorname{Ext}_{\Sigma_{r}}^{\mathrm{e}}\left(D^{\lambda}, D^{\mu}\right) \cong \operatorname{Hom}_{\Sigma_{r}}\left(\operatorname{rad} S^{\lambda}, D^{\mu}\right)
$$

ДокАЗАТЕЛЬСтво. Без ограничения обшности можно считать, что $h(\mu) \leqslant p$. Из того, что модуль $S^{\lambda}$ продолжаемьй, следует

$$
\operatorname{dim} \operatorname{Hom}_{\Sigma_{r}}\left(\operatorname{rad} S^{\lambda}, D^{\mu}\right) \leqslant \operatorname{dim} \operatorname{Ext}_{\Sigma_{r}}^{\mathrm{e}}\left(D^{\mu}, D^{\lambda}\right)
$$

Докажем обратное неравенство. Положим для краткости $\alpha=\left(\lambda^{t}\right)_{r}, \beta=\left(\mu^{t}\right)_{r}$ и $n=\operatorname{dim} \operatorname{Ext}_{\Sigma_{r}}^{\mathrm{e}}\left(D^{\mu}, D^{\lambda}\right)$. Пусть $M$ - некоторый продолжаемый $K \Sigma_{r}$-модуль такой, что $\operatorname{soc} M \cong D^{\lambda}$ и $M / \operatorname{soc} M \cong \bigoplus n D^{\mu}$, и $N-$ продолжение модуля $M$. Единственным не $p$-регулярным по столбцам набором $\nu \in \Lambda^{+}(r, r)$ таким, что $\nu_{1} \leqslant p$, может быть набор $\left(p^{r / p}, 0^{r-(r / p)}\right)$ и только в том случае, когда $r$ делится на $p$. Поэтому если $r$ не делится на $p$, то

$$
\operatorname{soc} N \cong L(\alpha), \quad N / \operatorname{soc} N \cong \bigoplus n L(\beta)
$$

Рассмотрим теперь случай, когда $r$ делится на $p$. Тогда модуль $N$ может содержать также композиционные факторы, изоморфные $L\left(p^{r / p}, 0^{r-(r / p)}\right)$.

Предположим сначала, что $\left(\beta_{1}, \ldots, \beta_{(r / p)-1}\right) \not\left(p^{(r / p)-1}\right)$. Тогда в силу следствия 1 получаем $\operatorname{Ext}_{S(r, r)}^{1}\left(L\left(p^{r / p}, 0^{r-(r / p)}\right), L(\beta)\right)=0$. 
Теперь предположим, что $\left(\beta_{1}, \ldots, \beta_{(r / p)-1}\right) \unrhd\left(p^{(r / p)-1}\right)$. Поскольку $\beta_{1} \leqslant p$, то $\beta_{1}=\cdots=\beta_{(r / p)-1}=p$. Если $\beta_{r / p} \leqslant p-2$, то по лемме 6 получаем

$$
\operatorname{Ext}_{S(r, r)}^{1}\left(L\left(p^{r / p}, 0^{r-(r / p)}\right), L(\beta)\right)=0 .
$$

$\mathrm{C}$ другой стороны, случай $\beta_{r / p}=p-1$ невозможен, так как тогда $\beta=\left(p^{r / p-1}\right.$, $\left.p-1,1,0^{r-(r / p)-1}\right) \unrhd \alpha$, что противоречит условию теоремы.

Таким образом, доказано, что в любом случае существуют подмодули $U \subset$ $V \subset N$ такие, что $\operatorname{soc}(V / U) \cong L(\alpha)$ и $(V / U) / \operatorname{soc}(V / U) \cong \bigoplus n L(\beta)$. Теперь можно заменить $N$ на $V / U$ и считать, что формула (1) выполнена.

В силу утверждения 3 получаем $\operatorname{dim} \operatorname{Hom}_{S(r, r)}(\operatorname{rad} \Delta(\alpha), L(\beta)) \geqslant n$. Умножая модули из этого неравенства на $e_{r}$, а затем тензорно на $\operatorname{sgn}_{r}$, получаем

$$
\operatorname{dim} \operatorname{Hom}_{\Sigma_{r}}\left(\operatorname{rad} S^{\lambda}, D^{\mu}\right) \geqslant n .
$$

СЛЕДСТВИЕ 2. Если $p>2 u \lambda, \mu-$ разбиения числа $r$ высоты менее $p$, то

$$
\operatorname{Ext}_{\Sigma_{r}}^{\mathrm{e}}\left(D^{\lambda}, D^{\mu}\right) \cong \operatorname{Ext}_{\Sigma_{r}}^{1}\left(D^{\lambda}, D^{\mu}\right)
$$

ДокАЗАТЕЛЬСТво. Поскольку $\operatorname{Ext}_{\Sigma_{r}}^{1}\left(D^{\lambda}, D^{\lambda}\right)=0$ (например, в силу утверждения 1 ), то $\lambda \neq \mu$. Следовательно, в силу леммы 4 без ограничения общности $\lambda \notin \mu$. Теперь требуемое следует из утверждения 1 и теоремы 1.

\section{§3. Неравенства}

Докажем следующую лемму общей теории модулей. Фиксируем некоторую $K$-алгебру $R$ и предположим, что все модули в следующей лемме $-K$-конечномерные $R$-модули.

Лемма 7. Пусть $P$ - некоторый модуль, $Q-$ его подмодуль, $0=Q_{0} \subset \cdots$ $\cdots \subset Q_{k}=Q-$ композиционный ряд и $P / Q \cong \bigoplus n L$, где $L-$ неприводимый модуль. Тогда существуют такие модули $P_{i}$ и неотрицательные целые числа $n_{i}$, где $i=0, \ldots, k, n_{0}+\cdots+n_{k}=n$, что имеют место следующие включения:

$$
\begin{aligned}
0= & Q_{0} \subset Q_{1} \subset \cdots \subset Q_{i-1} \subset Q_{i} \subset \cdots \subset Q_{k-1} \subset Q_{k} \\
& \cap \cap \cap \cap \cap \cap \\
& P_{0} \subset P_{1} \subset \cdots \subset P_{i-1} \subset P_{i} \subset \cdots \subset P_{k-1} \subset P_{k}=P
\end{aligned}
$$

и при этом $P_{i-1} \cap Q_{i}=Q_{i-1}, \quad \operatorname{soc}\left(P_{i} / P_{i-1}\right) \cong Q_{i} / Q_{i-1}, \quad\left(P_{i} / P_{i-1}\right) /$ $\operatorname{soc}\left(P_{i} / P_{i-1}\right) \cong \bigoplus n_{i} L \partial \Omega$ я $i=1, \ldots, k u P_{0} \cong \bigoplus n_{0} L$. 
ДокАЗАТЕЛЬСТво проведем индукцией по $k$. Случай $k=0$ очевиден. Предположим, что $k \geqslant 1$ и утверждение леммы верно для подмодулей длины менее $k$. Положим $P^{\prime}=P / Q_{k-1}$ и $Q^{\prime}=Q / Q_{k-1}$. Тогда $Q^{\prime}$ неприводим и $P^{\prime} / Q^{\prime} \cong \bigoplus n L$. Отсюда получаем, что $Q^{\prime} \subset \operatorname{soc} P^{\prime} \cong Q^{\prime} \oplus(\oplus m L)$ для некоторого $m \leqslant n$. Поэтому имеет место представление soc $P^{\prime}=Q^{\prime} \oplus Q^{\prime \prime}$, где $Q^{\prime \prime} \cong \bigoplus m L$.

Докажем, что $\operatorname{soc}\left(P^{\prime} / Q^{\prime \prime}\right)=Q^{\prime}+Q^{\prime \prime} / Q^{\prime \prime}$. Предположим противное. Тогда существует такой модуль $T$, что $Q^{\prime \prime} \subset T \subset P^{\prime}$ и $T / Q^{\prime \prime} \cong L$ и $T \neq Q^{\prime}+Q^{\prime \prime}$. Рассмотрим следующие два случая:

1) $T+Q^{\prime}=Q^{\prime \prime}+Q^{\prime}, T \cap Q^{\prime} / Q^{\prime \prime} \cap Q^{\prime} \cong L$;

2) $T+Q^{\prime} / Q^{\prime \prime}+Q^{\prime} \cong L, T \cap Q^{\prime}=Q^{\prime \prime} \cap Q^{\prime}$.

Первый случай невозможен, так как тогда $Q^{\prime \prime} \subset T \subset Q^{\prime}+Q^{\prime \prime}$ и, следовательно, $T=Q^{\prime}+Q^{\prime \prime}$. Поэтому реализуется второй случай. Тогда $T \cong T+Q^{\prime} / Q^{\prime} \subset P^{\prime} / Q^{\prime}$. Поскольку последний модуль полупрост, то модули $T$ и $T+Q^{\prime}$ также полупросты. По определению цоколя

$$
Q^{\prime} \oplus(\bigoplus(m+1) L) \cong T+Q^{\prime} \subset \operatorname{soc} P^{\prime} \cong Q^{\prime} \oplus(\bigoplus m L)
$$

Противоречие.

Положим теперь, что $n_{k}=n-m$ и модуль $P_{k-1}$ равен полному прообразу модуля $Q^{\prime \prime}$ при естественной проекции $P \rightarrow P / Q_{k-1}$. Теперь достаточно применить индуктивное предположение для модулей $Q_{k-1}, P_{k-1}$ и композиционного ряда $0=Q_{0} \subset \cdots \subset Q_{k-1}$.

Введем следующие обозначения. Пусть $\mu \vdash r$ и $\lambda \vdash r+1$ - некоторые $p$-регулярные разбиения. Положим

$$
\begin{aligned}
& \varepsilon(\lambda, \mu)= \begin{cases}1, & \text { если } \lambda=\mu^{B} \text { для некоторой } \mu \text {-конормальной клетки } B, \\
0 & \text { иначе },\end{cases} \\
& \gamma(\mu, \lambda)= \begin{cases}1, & \text { если } \mu=\lambda_{A} \text { для некоторой } \lambda \text {-нормальной клетки } A, \\
0 & \text { иначе. }\end{cases}
\end{aligned}
$$

ТЕОРема 2. Пусть $\lambda и \mu$-р-регулярные разбиения числа $r>1, \lambda \not \mu u$ A-н-хорошая клетка. Тогда

$$
\operatorname{dim} \operatorname{Ext}_{\Sigma_{r}}^{\mathrm{e}}\left(D^{\lambda}, D^{\mu}\right) \leqslant \sum_{\alpha \vdash r-1}\left[D^{\lambda} \downarrow_{\Sigma_{r-1}}: D^{\alpha}\right] \operatorname{dim} \operatorname{Ext}_{\Sigma_{r-1}}^{\mathrm{e}}\left(D^{\alpha}, D^{\mu_{A}}\right)+\varepsilon\left(\lambda, \mu_{A}\right) .
$$

ДокАЗАТЕЛЬСТВО. Без ограничения общности можно считать, что $h(\lambda) \leqslant p$. Положим для краткости $n=\operatorname{dim} \operatorname{Ext}_{\Sigma_{r}}^{\mathrm{e}}\left(D^{\lambda}, D^{\mu}\right)$. Тогда по теореме 1 получаем

$$
n=\operatorname{dim} \operatorname{Hom}_{\Sigma_{r}}\left(\operatorname{rad} S^{\lambda}, D^{\mu}\right)
$$

Пусть $V \subset \operatorname{rad} S^{\lambda}$ - такой $K \Sigma_{r}$-подмодуль, что $\operatorname{rad} S^{\lambda} / V \cong \bigoplus n D^{\mu}$. Пусть $M-$ $K \Sigma_{r}$-модуль, двойственный модулю $S^{\lambda} / V$. По леммам 1 и 2 модуль $\left(S^{\lambda} / V\right) \downarrow_{r-1}$ продолжаем. Так как модули $M \downarrow_{\Sigma_{r-1}}$ и $\left(S^{\lambda} / V\right) \downarrow_{\Sigma_{r-1}}$ тоже двойственны, то по лемме 1 модуль $M \downarrow_{\Sigma_{r-1}}$ продолжаем. 


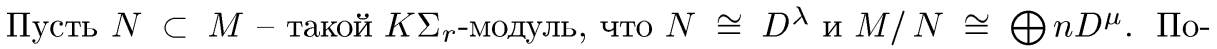
скольку $\operatorname{Hom}_{\Sigma_{r-1}}\left(D^{\mu_{A}}, D^{\mu} \downarrow_{\Sigma_{r-1}}\right) \neq 0$ в силу п. 2$)$ утверждения 2 , то сушествует $K \Sigma_{r-1}$-модуль $N^{\prime}$ такой, что $N \subset N^{\prime} \subset M$ и $N^{\prime} / N \cong \bigoplus n D^{\mu_{A}}$. Пусть $0=N_{0} \subset N_{1} \subset \cdots \subset N_{k}=N$ - некоторый $\Sigma_{r-1}$-композиционный ряд.

Применим теперь лемму 7 к случаю $R=K \Sigma_{r-1}, P=N^{\prime}, Q=N$ и $Q_{i}=N_{i}$. В силу определения 3 получаем оценки $n_{i} \leqslant \operatorname{dim} \operatorname{Ext}_{\Sigma_{r-1}}^{\mathrm{e}}\left(N_{i} / N_{i-1}, D^{\mu_{A}}\right), i=$ $1, \ldots, k$. Наконец, с учетом двойственности Фробениуса в силу п. 3 ) утверждения 2 имеем

$$
\begin{aligned}
n_{0} & \leqslant \operatorname{dim} \operatorname{Hom}_{\Sigma_{r-1}}\left(D^{\mu_{A}}, M \downarrow_{\Sigma_{r-1}}\right)=\operatorname{dim} \operatorname{Hom}_{\Sigma_{r-1}}\left(\left(S^{\lambda} / V\right) \downarrow_{\Sigma_{r-1}}, D^{\mu_{A}}\right) \\
& =\operatorname{dim} \operatorname{Hom}_{\Sigma_{r}}\left(S^{\lambda} / V, D^{\mu_{A} \uparrow^{\Sigma_{r}}}\right) \leqslant \operatorname{dim} \operatorname{Hom}_{\Sigma_{r}}\left(S^{\lambda}, D^{\mu_{A} \uparrow \Sigma_{r}}\right)=\varepsilon\left(\lambda, \mu_{A}\right) .
\end{aligned}
$$

СлЕДСТВИЕ 3. Пусть $p>2, \lambda u \mu-$ разбиения числа $r>1$ высоты менее $p$, $\lambda \not \mu и A-\mu$-хорошая клетка. Тогда

$$
\operatorname{dim} \operatorname{Ext}_{\Sigma_{r}}^{1}\left(D^{\lambda}, D^{\mu}\right) \leqslant \sum_{\alpha \vdash r-1}\left[D^{\lambda} \downarrow_{\Sigma_{r-1}}: D^{\alpha}\right] \operatorname{dim} \operatorname{Ext}_{\Sigma_{r-1}}^{1}\left(D^{\alpha}, D^{\mu_{A}}\right)+\varepsilon\left(\lambda, \mu_{A}\right) .
$$

ДокАЗАТЕЛЬСТво. В силу утверждения 1 достаточно считать, что $\mu \neq \lambda$. Теперь требуемое неравенство следует из следствия 2 и теоремы 2.

Теорема 3. Пусть $\lambda u \mu-p$-регулярные разбиения числа $r, \lambda \notin \mu u B-$ $\mu$-кохорошая клетка. Тогда

$$
\begin{aligned}
& \operatorname{dim} \operatorname{Ext}_{\Sigma_{r}}^{\mathrm{e}}\left(D^{\lambda}, D^{\mu}\right) \leqslant \sum_{\substack{\alpha \vdash r+1 \\
h(\alpha) \leqslant p}}\left[D^{\lambda \uparrow \Sigma_{r+1}}: D^{\alpha}\right] \operatorname{dim} \operatorname{Ext}_{\Sigma_{r+1}}^{\mathrm{e}}\left(D^{\alpha}, D^{\mu^{B}}\right) \\
& +\delta_{h(\lambda), p}\left[S^{(\lambda, 1)}: D^{\mu^{B}}\right]+\gamma\left(\lambda, \mu^{B}\right) .
\end{aligned}
$$

ДоКАЗАТЕЛЬСТво. Без ограничения обшности можно считать, что $h(\lambda) \leqslant p$. Положим для краткости $m=\operatorname{dim} \operatorname{Ext}_{\Sigma_{r}}\left(D^{\lambda}, D^{\mu}\right)$. Тогда по теореме 1 получаем

$$
m=\operatorname{dim} \operatorname{Hom}_{\Sigma_{r}}\left(\operatorname{rad} S^{\lambda}, D^{\mu}\right) .
$$

Пусть $V \subset \operatorname{rad} S^{\lambda}$ - такой $K \Sigma_{r}$-подмодуль, что $\operatorname{rad} S^{\lambda} / V \cong \bigoplus m D^{\mu}$. Пусть $M-$ $K \Sigma_{r}$-модуль, двойственный модулю $S^{\lambda} / V$.

Выберем $K \Sigma_{r+1}$-подмодуль $T$ модуля $M \uparrow^{\Sigma_{r+1}}$ следуюшим образом. Если $h(\lambda)<p$, то положим $Q=M \uparrow^{\Sigma_{r+1}}$. Предположим, что $h(\lambda)=p$. По лемме 3 сушествует подмодуль $U$ модуля $S^{\lambda} \uparrow^{\Sigma_{r+1}}$ такой, что $U \cong S^{(\lambda, 1)}$ и $S^{\lambda} \uparrow^{\Sigma_{r+1}} / U$ продолжаем. По лемме 1 получаем, что модуль $S^{\lambda} \uparrow_{r+1} / U+V \uparrow \Sigma_{r+1}$, а следовательно, и изоморфный ему модуль $\left(S^{\lambda} \uparrow \Sigma_{r+1} / V \uparrow \Sigma_{r+1}\right) /\left(U+V \uparrow \Sigma_{r+1} / V \uparrow \Sigma_{r+1}\right)$ продолжаемы. Так как $S^{\lambda} \uparrow^{\Sigma_{r+1}} / V \uparrow \Sigma_{r+1}$ двойствен модулю $M \uparrow \Sigma_{r+1}$ и

$$
U+V \uparrow^{\Sigma_{r+1}} / V \uparrow^{\Sigma_{r+1}} \cong U / U \cap V \uparrow^{\Sigma_{r+1}}
$$

то мы получаем, что сушествует продолжаемый подмодуль $T$ модуля $M \uparrow \Sigma_{r+1}$ такой, что $M \uparrow^{\Sigma_{r+1}} / T$ - гомоморфный образ модуля $S^{(\lambda, 1)}$. 
Итак, модуль $T$ в обоих случаях выбран. При этом $T$ продолжаем и выполнено неравенство

$$
\left[M \uparrow^{\Sigma_{r+1}} / T: D^{\mu^{B}}\right] \leqslant \delta_{h(\lambda), p}\left[S^{(\lambda, 1)}: D^{\mu^{B}}\right] .
$$

Пусть $N \subset M \uparrow^{\Sigma_{r+1}}$ - такой $K \Sigma_{r+1}$-подмодуль, что $N \cong D^{\lambda \uparrow \Sigma_{r+1}}$ и $M \uparrow \Sigma^{\Sigma_{r+1}}$ / $N \cong \bigoplus m D^{\mu \uparrow \Sigma_{r+1}}$.

Поскольку $\operatorname{Hom}_{\Sigma_{r+1}}\left(D^{\mu^{B}}, D^{\mu \uparrow \Sigma_{r+1}}\right) \neq 0$ в силу п. 4) утверждения 2 , то сушествует $K \Sigma_{r+1}$-модуль $N^{\prime}$ такой, что $N \subset N^{\prime} \subset M \uparrow \Sigma_{r+1}$ и $N^{\prime} / N \cong \bigoplus m D^{\mu^{B}}$.

Имеем $N^{\prime} \cap T / N \cap T \cong \bigoplus n D^{\mu^{B}}$, где $n=m$ в случае $h(\lambda)<p$ и $n \geqslant m-$ $\left[S^{(\lambda, 1)}: D^{\mu^{B}}\right]$ в случае $h(\lambda)=p$. Пусть $0=T_{0} \subset T_{1} \subset \cdots \subset T_{k}=N \cap T-$ некоторый $\Sigma_{r+1}$-композиционный ряд. Так как модуль $N \cap T$ продолжаем, то для любого $i=1, \ldots, k$ высота разбиения $\alpha$, где $D^{\alpha} \cong T_{i} / T_{i-1}$, не более $p$.

Применим теперь лемму 7 к случаю $R=K \Sigma_{r+1}, P=N^{\prime} \cap T, Q=N \cap T$ и $Q_{i}=T_{i}$. В силу определения 3 получаем оценки $n_{i} \leqslant \operatorname{dim} \operatorname{Ext}_{\Sigma_{r+1}}^{\mathrm{e}}\left(T_{i} / T_{i-1}, D^{\mu^{B}}\right)$, $i=1, \ldots, k$. Наконец, с учетом двойственности Фробениуса в силу п. 1$)$ утверждения 2 имеем

$$
\begin{aligned}
n_{0} & \leqslant \operatorname{dim} \operatorname{Hom}_{\Sigma_{r+1}}\left(D^{\mu^{B}}, M \uparrow \Sigma_{r+1}\right)=\operatorname{dim} \operatorname{Hom}_{\Sigma_{r+1}}\left(\left(S^{\lambda} / V\right) \uparrow \Sigma_{r+1}, D^{\mu^{B}}\right) \\
& =\operatorname{dim} \operatorname{Hom}_{\Sigma_{r}}\left(S^{\lambda} / V, D^{\mu^{B}} \downarrow_{\Sigma_{r}}\right) \leqslant \operatorname{dim} \operatorname{Hom}_{\Sigma_{r}}\left(S^{\lambda}, D^{\mu^{B}} \downarrow_{\Sigma_{r}}\right)=\gamma\left(\lambda, \mu^{B}\right) .
\end{aligned}
$$

СЛЕДСТВИЕ 4. Пусть $p>2, \lambda u \mu$-разбиения числа $r$ высоты менее $p$, $\lambda \not \mu и B-\mu$-кохорошая клетка. Тогда

$$
\operatorname{dim} \operatorname{Ext}_{\Sigma_{r}}^{1}\left(D^{\lambda}, D^{\mu}\right) \leqslant \sum_{\alpha \vdash r+1}\left[D^{\lambda \uparrow \Sigma_{r+1}}: D^{\alpha}\right] \operatorname{dim} \operatorname{Ext}_{\Sigma_{r+1}}^{1}\left(D^{\alpha}, D^{\mu^{B}}\right)+\gamma\left(\lambda, \mu^{B}\right)
$$

ДоКАЗАТЕЛЬство следует из теоремы 3 аналогично доказательству следствия 3.

Сделаем следуюшее предположение: полученные неравенства позволяют вычислить размерности пространств $\operatorname{Hom}_{\Sigma_{r}}\left(\operatorname{rad} S^{\lambda}, D^{\mu}\right)$, где $h(\lambda) \leqslant p$, для малых $r$ (в пределах известных композиционных матрищ).

Возможно также доказать аналогичные неравенства для размерностей $\operatorname{Ext}_{\Sigma_{r}}^{1}\left(D^{\lambda}, D^{\mu}\right)$, когда $\lambda$ и $\mu$-произвольные $p$-регулярные разбиения числа $r$. Однако в этом случае функции $\varepsilon$ и $\gamma$ придется заменить на менее удобные для вычисления.

ТЕОРЕМА 4. Пусть $\lambda и \mu$-различные р-регулярнье разбиения числа $r>1$ и А-н-хорошая клетка. Тогда

$$
\begin{aligned}
\operatorname{dim} \operatorname{Ext}_{\Sigma_{r}}^{1}\left(D^{\lambda}, D^{\mu}\right) \leqslant & \sum_{\alpha \vdash r-1}\left[D^{\lambda} \downarrow_{\Sigma_{r-1}}: D^{\alpha}\right] \operatorname{dim} \operatorname{Ext}_{\Sigma_{r-1}}^{1}\left(D^{\alpha}, D^{\mu_{A}}\right) \\
& +\operatorname{dim} \operatorname{Hom}_{\Sigma_{r}}\left(\operatorname{rad} D^{\mu_{A} \uparrow \Sigma_{r}}, D^{\mu}\right) .
\end{aligned}
$$


ДокАЗАТЕльСТво аналогично доказательству теоремы 2, с тем отличием, что вместо $M$ необходимо рассмотреть любой $K \Sigma_{r}$-модуль $C$ такой, что $\operatorname{soc} C \cong D^{\lambda}$ и $C / \operatorname{soc} C \cong \bigoplus \operatorname{dim} \operatorname{Ext}_{\Sigma_{r}}^{1}\left(D^{\lambda}, D^{\mu}\right) D^{\mu}$. Достаточно считать, что $\lambda \sim \mu$. Оценка соответствующего $n_{0}$ теперь имеет следующий вид:

$$
\begin{aligned}
n_{0} & \leqslant \operatorname{dim} \operatorname{Hom}_{\Sigma_{r-1}}\left(D^{\mu_{A}}, C \downarrow \Sigma_{r-1}\right)=\operatorname{dim} \operatorname{Hom}_{\Sigma_{r}}\left(D^{\mu_{A} \uparrow \Sigma_{r}}, C\right) \\
& =\operatorname{dim}_{\operatorname{Hom}_{\Sigma_{r}}}(J, C) \leqslant \operatorname{dim}_{\operatorname{Hom}_{\Sigma_{r}}}\left(\operatorname{rad} J, D^{\lambda}\right)=\operatorname{dim}_{\operatorname{Hom}_{\Sigma_{r}}}\left(\operatorname{rad} D^{\mu_{A} \uparrow \Sigma_{r}}, D^{\lambda}\right),
\end{aligned}
$$

где $J$ - неразложимое прямое слагаемое модуля $D^{\mu_{A}} \uparrow^{\Sigma_{r}}$ из того же блока, что и $D^{\mu}$ (т.е. $\left.J / \operatorname{rad} J \cong D^{\mu}\right)$.

ТЕОРемА 5. Пусть $\lambda и \mu$-различные р-регулярные разбиения числа $r$ и $B$ $\mu$-кохорошая клетка. Тогда

$$
\begin{aligned}
\operatorname{dim} \operatorname{Ext}_{\Sigma_{r}}^{1}\left(D^{\lambda}, D^{\mu}\right) \leqslant & \sum_{\alpha \vdash r+1}\left[D^{\lambda \uparrow \Sigma_{r+1}}: D^{\alpha}\right] \operatorname{dim} \operatorname{Ext}_{\Sigma_{r+1}}^{1}\left(D^{\alpha}, D^{\mu^{B}}\right) \\
& +\operatorname{dim} \operatorname{Hom}_{\Sigma_{r}}\left(\operatorname{rad} D^{\mu^{B}} \downarrow_{\Sigma_{r}}, D^{\lambda}\right) .
\end{aligned}
$$

ДоКАЗАТЕЛЬСТво аналогично доказательству теоремы 3 , с тем отличием, что вместо $M$ необходимо рассмотреть любой $K \Sigma_{r}$-модуль $C$ такой, что $\operatorname{soc} C \cong D^{\lambda}$ и $C / \operatorname{soc} C \cong \bigoplus \operatorname{dim} \operatorname{Ext}_{\Sigma_{r}}^{1}\left(D^{\lambda}, D^{\mu}\right) D^{\mu}$. В данном случае нам не важна продолжаемость модулей и нет необходимости выбирать продолжаемьй подмодуль в $C$. Достаточно считать, что $\lambda \sim \mu$. Оценка соответствующего $n_{0}$ теперь имеет следующий вид:

$$
\begin{aligned}
n_{0} & \leqslant \operatorname{dim} \operatorname{Hom}_{\Sigma_{r+1}}\left(D^{\mu^{B}}, C \uparrow \Sigma_{r+1}\right)=\operatorname{dim} \operatorname{Hom}_{\Sigma_{r}}\left(D^{\mu^{B}} \downarrow_{\Sigma_{r}}, C\right) \\
& =\operatorname{dim} \operatorname{Hom}_{\Sigma_{r}}(I, C) \leqslant \operatorname{dim}_{\operatorname{Hom}_{\Sigma_{r}}}\left(\operatorname{rad} I, D^{\lambda}\right)=\operatorname{dim} \operatorname{Hom}_{\Sigma_{r}}\left(\operatorname{rad} D^{\mu^{B}} \downarrow_{\Sigma_{r}}, D^{\lambda}\right),
\end{aligned}
$$

где $I$ - неразложимое прямое слагаемое модуля $D^{\mu^{B}} \downarrow_{\Sigma_{r}}$ из того же блока, что и $D^{\mu}$ (т.е. $\left.I / \operatorname{rad} I \cong D^{\mu}\right)$.

ЗАмЕчАнИЕ 3. Несмотря на то, что в общем случае размерности пространств $\operatorname{Hom}_{\Sigma_{r}}\left(\operatorname{rad} D^{\mu} \uparrow^{\Sigma_{r}}, D^{\lambda}\right)$ и $\operatorname{Hom}_{\Sigma_{r}}\left(\operatorname{rad} D^{\mu^{B}} \downarrow_{\Sigma_{r}}, D^{\lambda}\right)$ неизвестны, с использованием результатов работы [4] иногда можно заключить, что они нулевые.

\section{§4. Формула для $\mathrm{Ext}^{1}$}

Для краткости разбиение $\lambda$, для которого модуль $D^{\lambda}$ вполне расщепляемый, будем называть также вполне расщепляемым. Клетка $A$ называется граничной клеткой разбиения $\lambda$, если $A \in[\lambda]$ и $A+(1,1) \notin[\lambda]$. Для пары чисел $i$ и $j$ обозначим через $R_{i, j}(\lambda)$ множество граничных клеток $(x, y)$ диаграммы $[\lambda]$ таких, что $x \geqslant i$ и $y \geqslant j$, и положим $h_{i, j}(\lambda)=\left|R_{i, j}(\lambda)\right|$. Множество $R_{i, j}(\lambda)$ называется косы.м крюком и в случае $h_{i, j}(\lambda)=p$ также косым $p$-крюком. Положим $\sum \lambda=\sum_{i=1}^{h(\lambda)} \lambda_{i}$.

ОПРЕДЕЛЕНИЕ 4. Разбиение $\lambda$ назовем больиим, если оно вполне расщепляемое высоты более 1 и $h_{1,1}(\lambda) \geqslant p$. В этом случае обозначим через $\tilde{\lambda}$ разбиение, диаграмма которого получается из $[\lambda]$ перемешением отрезка $(h, j), \ldots,\left(h, \lambda_{h}\right)$ в 
первую строку, где $(h, j)$ - самая правая клетка последней строки диаграммы $[\lambda]$ вычета $\operatorname{res}\left(1, \lambda_{1}+1\right)$.

Легко видеть, что если $\lambda_{A}$ - большое разбиение, то $\lambda$ - также большое разбиение. Докажем теперь лемму о том, как связаны операция $\lambda \mapsto \tilde{\lambda}$ и операция удаления клетки.

ЛЕмма 8. Пусть $\lambda$ - большое разбиение высоты . Пусть $F$ и $\widetilde{F}-$ самые правые клетки первых строк диаграмм $[\lambda] u[\tilde{\lambda}]$ соответственно, $L u \tilde{L}-$ самые правые клетки последних строк диаграм⿻ $[\lambda]$ и $[\tilde{\lambda}]$ соответственно $u A$ - некоторая клетка такая, что $1<r(A)<$. Тогда выполнены следующие утверждения.

1) Имеем $h_{1, \lambda_{h}}(\tilde{\lambda})=p u R_{1, \tilde{\lambda}_{h-1}}(\tilde{\lambda}) \subset R_{1, \lambda_{h}}(\tilde{\lambda})$.

2) Клетка $\widetilde{F}$ всегда $\tilde{\lambda}$-хорошая; $\chi(\lambda)<p$ тогда и только тогда, когда $L-\lambda$-хорошая клетка. При выполнении последних условий не существует $\tilde{\lambda}$-добавляемых клеток $C$ таких, что res $C=\operatorname{res} \widetilde{F}$ u $r(C) \leqslant h$, разбиение $\lambda_{L}$ большое и $\widetilde{\lambda_{L}}=\tilde{\lambda}_{\widetilde{F}}$. Ecли $\chi(\lambda)=p, \operatorname{mo} \varepsilon\left(\lambda, \tilde{\lambda}_{\widetilde{F}}\right)=1$.

3) Предположим, что $h(\tilde{\lambda})=h$. Тогда $F-\lambda$-хорошая клетка, если и только если $\tilde{L}-\tilde{\lambda}$-хорошая клетка. При выполнении последних условий не существует $\tilde{\lambda}$-добавляемых клеток $C$ таких, что $\operatorname{res} C=\operatorname{res} F$ u $r(C)<h$, разбиение $\lambda_{F}$ больиое и $\widetilde{\lambda_{F}}=\tilde{\lambda}_{\tilde{L}}$.

4) Клетка $A-\lambda$-хорошая клетка тогда и только тогда, когда $A-\tilde{\lambda}$-хо-

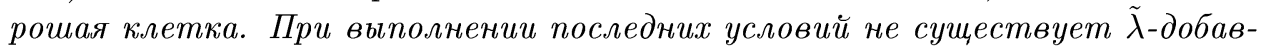
ляемых клеток $C$ таких, что $\operatorname{res} C=\operatorname{res} A$ u $r(C) \leqslant h$, разбиение $\lambda_{A}$ большое $u \widetilde{\lambda_{A}}=\tilde{\lambda}_{A}$.

ДокАЗАТЕльство. Пусть $(h, j)$ - самая правая клетка последней строки диаграммы $[\lambda]$ вычета $\operatorname{res}\left(1, \lambda_{1}+1\right)$. Справедливо следующее утверждение: любая $\tilde{\lambda}$-добавляемая клетка остатка $a$, лежашая в первых $h-1$ строках, имеет вид $B+(0,1)$, где $B \in R_{1, \tilde{\lambda}_{h-1}}(\tilde{\lambda})$ и res $B=a-1$.

1) Из определения 4 имеем, что $R_{1, \lambda_{h}}(\tilde{\lambda})$ получается из косого $p$-крюка $R_{1, j}(\lambda)$ перемещением всех клеток последней строки в первую. Отсюда $h_{1, \lambda_{h}}(\tilde{\lambda})=$ $h_{1, j}(\lambda)=p$. Включение следует из неравенств $\tilde{\lambda}_{h-1} \geqslant \lambda_{h-1} \geqslant \lambda_{h}$.

2 ) Единственной клеткой из $R_{1, \lambda_{h}}(\tilde{\lambda})$ вычета res $\widetilde{F}$ является клетка $\widetilde{F}$. В случае $h(\tilde{\lambda})=h$ получаем res $\tilde{L}=\operatorname{res} F \neq \operatorname{res} \widetilde{F}$. Следовательно, $\widetilde{F}$ - единственная $\tilde{\lambda}$-удаляемая клетка вычета res $\widetilde{F}$, и эта клетка $\tilde{\lambda}$-хорошая. Второе утверждение следует из [16]. Единственной клеткой из $R_{1, \lambda_{h}}(\tilde{\lambda})$ вычета res $\widetilde{F}-1$ является клетка $\widetilde{F}-(0,1)$. Поскольку клетка $\widetilde{F}$ не $\tilde{\lambda}$-добавляемая, то в силу утверждения, сфороулированного в начале доказательства для $a=\operatorname{res} \widetilde{F}$, в первой $h-1$ строке нет $\tilde{\lambda}$-добавляемых клеток вычета res $\widetilde{F}$. Предположим теперь, что $\chi(\lambda)<p$. В этом случае при переходе к $\tilde{\lambda}$ происходит перемещение по крайней мере двух клеток (т.е. $j<\lambda_{h}$ ) и, следовательно, $F+(0,1) \neq \widetilde{F}$. Так как обе эти клетки принадлежат косому $p$-крюку $R_{1, \lambda_{h}}(\tilde{\lambda})$, то res $F+1 \neq \operatorname{res} \widetilde{F}$. Из определения 4 следует, что $(h, j)-\tilde{\lambda}$-добавляемая клетка строки $h$ и она имеет вычет res $F+1$. Следовательно, и в строке $h$ нет $\tilde{\lambda}$-добавляемой клетки вычета res $\widetilde{F}$.

Равенство $\widetilde{\lambda_{L}}=\tilde{\lambda}_{\widetilde{F}}$ получается перемещением отрезка $(h, j), \ldots,\left(h, \lambda_{h}-1\right)$ из последней строки диаграммы $\left[\lambda_{L}\right]$ в первую. Если $\chi(\lambda)=p$, то из определения 4 
следует, что $(h, j)=L$ и $\tilde{\lambda}=\left(\lambda_{L}\right)^{\widetilde{F}}$. Отсюда $\lambda=\left(\tilde{\lambda}_{\widetilde{F}}\right)^{L}$. Клетка $L$ является $\tilde{\lambda}_{\widetilde{F}}$-конормальной, так как ниже нее нет ни одной удаляемой клетки диаграммы $\left[\tilde{\lambda}_{\widetilde{F}}\right]$. Следовательно, $\varepsilon\left(\lambda, \tilde{\lambda}_{\widetilde{F}}\right)=1$.

$3)$ Легко видеть, что $\operatorname{res} \tilde{L}=\operatorname{res} F$. Если $F-\lambda$-хорошая клетка, то $F-(0,1)-$ единственная клетка из $R_{1, \lambda_{h}}(\tilde{\lambda})$ вычета $\operatorname{res} F-1$. Так как клетка $F$ не $\tilde{\lambda}$-добавляемая, то в силу утверждения, сформулированного в начале доказательства для $a=\operatorname{res} F$, в первых $h-1$ строках нет $\tilde{\lambda}$-добавляемых клеток вычета $\operatorname{res} F$. Следовательно, $\tilde{L}-\tilde{\lambda}$-хорошая клетка.

Предположим теперь, что $F$ - не $\lambda$-хорошая клетка. Так как $\lambda$ вполне расшепляемо, то отсюда следует, что $\lambda_{1}=\lambda_{2}$. Рассмотрим следуюшие случаи.

a) $h>2$. Тогда $F+(1,1)-\tilde{\lambda}$-добавляемая клетка вычета $\operatorname{res} F$. Клетка $F-$ единственная из $R_{1, \lambda_{n}}(\tilde{\lambda})$ вычета res $F$. Однако $F$ - не $\tilde{\lambda}$-удаляемая клетка. Следовательно, не существует $\tilde{\lambda}$-удаляемых клеток вычета res $F$, расположенных выше $\tilde{L}$, и эта клетка не $\tilde{\lambda}$-хорошая.

б) $h=2$. Тогда $\widetilde{F}+(0,1)-\tilde{\lambda}$-добавляемая клетка вычета res $F$ и $\tilde{L}-$ не $\tilde{\lambda}$-хорошая клетка.

Равенство $\widetilde{\lambda_{F}}=\tilde{\lambda}_{\tilde{L}}$ получается перемешением отрезка $(h, j-1), \ldots,\left(h, \lambda_{h}\right)$ из последней строки диаграммы $\left[\lambda_{F}\right]$ в первую.

4) Предположим, что $A-\lambda$-хорошая клетка. Если $r(A)<h-1$, то вьше $A$ находится не более $p-2$ граничных клеток диаграммы $[\tilde{\lambda}]$. Следовательно, выше $A$ не лежит ни одной $\tilde{\lambda}$-добавляемой клетки вычета $\operatorname{res} A$. Если $A$ лежит в строке $h-1$, то $\lambda_{h}<\lambda_{h-1}$. Отсюда $h_{1, \tilde{\lambda}_{h-1}}<p$ и по той же причине вьше $A$ не лежит ни одной $\tilde{\lambda}$-добавляемой клетки вычета res $A$. В обоих случаях $A-\tilde{\lambda}$-хорошая клетка.

Предположим теперь, что $A-\tilde{\lambda}$-хорошая клетка. Если $A$ не лежит в строке $h-1$, то она $\lambda$-удаляемая. Если $A$ лежит в строке $h-1$, то $h_{1, \tilde{\lambda}_{h-1}}<p$ и $\lambda_{h}<\lambda_{h-1}$. Поэтому $A$ - снова $\lambda$-удаляемая клетка. В обоих случаях $A-\lambda$-хорошая клетка.

Из предыдуших рассуждений следует, что если $A-\tilde{\lambda}$-хорошая клетка, то клетка $A-(0,1)$ принадлежит косому $p$-крюку $R_{1, \lambda_{h}}(\tilde{\lambda})$ и, таким образом, является единственной клеткой из $R_{1, \lambda_{h}}(\tilde{\lambda})$ вычета $\operatorname{res} A-1$. Поскольку клетка $A$ не $\tilde{\lambda}$-добавляемая, то в силу утверждения, сформулированного в начале доказательства для $a=\operatorname{res} A$, в первых $h-1$ строках нет $\tilde{\lambda}$-добавляемых клеток вычета $\operatorname{res} A$. Так как $A \neq F+(0,1)$ и обе эти клетки принадлежат косому $p$-крюку $R_{1, \lambda_{h}}(\tilde{\lambda})$, то $\operatorname{res} A \neq \operatorname{res} F+1 ; \tilde{\lambda}$-добавляемой клеткой строки $h$ является клетка $(h, j)$, и она имеет вычет res $F+1$. Следовательно, в строке $h$ нет $\tilde{\lambda}$-добавляемой клетки вычета res $A$.

Равенство $\widetilde{\lambda_{A}}=\tilde{\lambda}_{A}$ получается перемешением отрезка $(h, j), \ldots,\left(h, \lambda_{h}\right)$ из последней строки диаграммы $\left[\lambda_{A}\right]$ в первую.

ПримеР. Пусть $p=7, \lambda=(5,4,4,3)$ и $h=4$,

$$
\begin{aligned}
& \times \times 34 \times \times \times 3456
\end{aligned}
$$

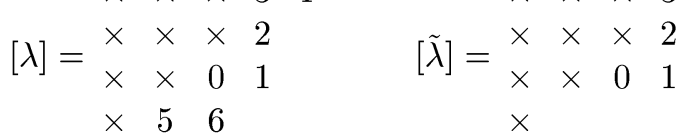

Тогда $j=2$ и $\tilde{\lambda}=(7,4,4,1)$. В левой диаграмме вычетами отмечено множество $R_{1, j}(\lambda)$, а в правой - множество $R_{1, \lambda_{h}}(\tilde{\lambda})$. 
Определим по индукции множества $X_{r}, r \in \mathbb{N}$, пар разбиений. Положим $X_{1}=\varnothing$. Предположим, что $r>1$ и множества $X_{1}, \ldots, X_{r-1}$ уже построены. Пару разбиений $(\lambda, \mu)$ числа $r$ включаем в $X_{r}$, если выполнены следующие условия:

1) $\lambda$ вполне расшепляемо, $\lambda \triangleleft \mu$ и $\lambda \sim \mu$;

2 ) для любой $\mu$-хорошей клетки $A$ либо $\varepsilon\left(\lambda, \mu_{A}\right)=1$, либо сушествует $\lambda$-хорошая клетка $B$ такая, что $\operatorname{res} A=\operatorname{res} B$ и $\left(\lambda_{B}, \mu_{A}\right) \in X_{r-1}$.

Положим $X=\bigcup_{r=1}^{\infty} X_{r}$.

Лемма 9. Пара разбиений $(\lambda, \mu)$ принадлежит множеству $X$ тогда и только тогда, когда $\lambda$ - больиое разбиение $и \mu=\tilde{\lambda}$.

ДокАЗАТЕЛЬСтво. Проведем индукцию по $\sum \lambda$. Если $\sum \lambda=1$, то $\lambda=(1)$ и $\lambda-$ не большое разбиение. Предположим теперь, что $\sum \lambda=r>1$ и лемма выполнена для пар разбиений с суммой менњше $r$. В доказательстве будем полагать, что клетки $F, L$ связаны с $\lambda$, а в случае, когда $\lambda$ - большое разбиение, то связаны также и клетки $\widetilde{F}, \tilde{L}$, как в лемме 8 .

Предположим сначала, что $\lambda$ - большое разбиение высоты $h$. Из непосредственной конструкции разбиения $\tilde{\lambda}$ видно, что для пары $(\lambda, \mu)$, где $\mu=\tilde{\lambda}$, условие 1$)$ вьполнено.

Проверим условие 2 ). Пусть $A$ - некоторая $\tilde{\lambda}$-хорошая клетка.

Рассмотрим сначала случай $A=\widetilde{F}$. Если $\chi(\lambda)=p$, то в силу п. 2) леммы 8 получаем $\varepsilon\left(\lambda, \tilde{\lambda}_{A}\right)=1$. Если $\chi(\lambda)<p$, то в силу п. 2$)$ леммы 8 получаем, что $L-$ $\lambda$-хорошая клетка, $\lambda_{L}$ - большое разбиение и $\widetilde{\lambda_{L}}=\tilde{\lambda}_{A}$. Отсюда по предположению индукции $\left(\lambda_{L}, \tilde{\lambda}_{A}\right) \in X_{r-1}$.

Пусть теперь $h(\tilde{\lambda})=h$ и $A=\tilde{L}$. В силу п.3) леммы 8 получаем, что $F-$ $\lambda$-хорошая клетка, $\lambda_{F}$-большое разбиение и $\widetilde{\lambda_{F}}=\tilde{\lambda}_{A}$. Отсюда по предположению индукщии $\left(\lambda_{F}, \tilde{\lambda}_{A}\right) \in X_{r-1}$.

Наконец, если $1<r(A)<h$, то в силу п. 4) леммы 8 получаем, что $A-\lambda$-хорошая клетка, $\lambda_{A}-$ большое разбиение и $\widetilde{\lambda_{A}}=\tilde{\lambda}_{A}$. Отсюда по предположению индукции $\left(\lambda_{A}, \tilde{\lambda}_{A}\right) \in X_{r-1}$.

Рассмотрев все возможные случаи, мы получили в силу определения множества $X_{r}$, что $(\lambda, \tilde{\lambda}) \in X_{r}$.

Пусть теперь, наоборот, $(\lambda, \mu) \in X_{r}$. Для дальнейших рассуждений будем иметь в виду, что если $A \neq B, B-\nu$-удаляемая клетка и $A-\nu_{B}$-добавляемая клетка, то $A-\nu$-добавляемая клетка.

Предположим сначала, что $\varepsilon\left(\lambda, \mu_{A}\right)=1$ для некоторой $\mu$-хорошей клетки $A$. Тогда $\lambda=\left(\mu_{A}\right)^{B}$, где $B$ - некоторая $\mu$-добавляемая клетка вычета res $A$. Поскольку $\lambda \triangleleft \mu$, то $B$ расположена строго ниже, чем $A$. Отсюда получаем, что $B-$ $\lambda$-удаляемая клетка, $A-\lambda_{B}$-добавляемая, а следовательно, и $\lambda$-добавляемая клетка. Однако такое возможно только при $\chi(\lambda)=p, A=F+(0,1)$ и $B=L$. По определению 4 тогда получаем $\mu=\tilde{\lambda}$.

Теперь рассмотрим случай, когда $\varepsilon\left(\lambda, \mu_{A}\right)=0$ для любой $\mu$-хорошей клетки $A$. Возьмем некоторую $\mu$-хорошую клетку $A$. Понятно, что такая клетка всегда существует, и так как $\mu \triangleright \lambda$, то она находится в первых $h$ строках. В силу сделанного предположения о значении функции $\varepsilon$ существует некоторая $\lambda$-хорошая клетка $B$ такая, что $\left(\lambda_{B}, \mu_{A}\right) \in X_{r-1}$. По предположению индукции получаем, что $\lambda_{B}-$ большое разбиение и $\widetilde{\lambda_{B}}=\mu_{A}$. 
Рассмотрим следующие случаи.

a) $B=F$ и $h(\tilde{\lambda})=h$. В силу п. 3$)$ леммы 8 получаем $\widetilde{\lambda_{B}}=\tilde{\lambda}_{\tilde{L}}=\mu_{A}$ и, следовательно, $A-\tilde{\lambda}_{\tilde{L}}$-добавляемая клетка. Если предположить, что $r(A)<h$, то эта клетка $A$ также $\tilde{\lambda}$-добавляемая. Однако $\lambda \sim \mu$ и res $A=\operatorname{res} \tilde{L}=\operatorname{res} F$. Это противоречит п. 3 ) леммы 8. Поэтому $A$ находится в строке $h$ и $A=\tilde{L}$. Отсюда $\mu=\tilde{\lambda}$.

б) $B=F$ и $h(\tilde{\lambda})<h$. Легко видеть, что в этом случае $\lambda_{B}-$ не большое разбиение. Поэтому этот случай не реализуется.

в) $B=L$. В силу п. 2) леммы 8 получаем $\chi(\lambda)<p$ и $\widetilde{\lambda_{B}}=\tilde{\lambda}_{\widetilde{F}}=\mu_{A}$ и, следовательно, $A-\tilde{\lambda}_{\widetilde{F}}$-добавляемая клетка. Если предположить, что $A$ расположена на первой строке, то эта клетка также $\tilde{\lambda}$-добавляемая. Однако $\lambda \sim \mu$ и res $A=\operatorname{res} \widetilde{F}$. Это противоречит п. 2) леммы 8. Поэтому $A$ находится в первой строке и $A=\widetilde{F}$. Отсюда $\mu=\tilde{\lambda}$.

г) $1<r(B)<h$. В силу п. 4$)$ леммы 8 получаем, что $B-\tilde{\lambda}$-хорошая и $\widetilde{\lambda_{B}}=$ $\tilde{\lambda}_{B}=\mu_{A}$. Если предположить, что $A \neq B$, то $A$ - также $\tilde{\lambda}$-добавляемая клетка. Однако $\lambda \sim \mu$ и $\operatorname{res} A=\operatorname{res} B$. Это противоречит п. 4) леммы 8. Поэтому $A=B$ и $\mu=\tilde{\lambda}$.

Следствие 3 для вполне расщепляемых разбиений принимает следующий вид.

СлеДСТВИЕ 5. Пусть $p>2 u \lambda, \mu-$ разбиения числа $r>1$ высоты менее $p$ такие, что $\lambda$ вполне расщепляемо, $\lambda \not \mu, \lambda \sim \mu, u A-\mu$-хорошая клетка. Тогда $\operatorname{dim} \operatorname{Ext}_{\Sigma_{r}}^{1}\left(D^{\lambda}, D^{\mu}\right) \leqslant \operatorname{dim} \operatorname{Ext}_{\Sigma_{r-1}}^{1}\left(D^{\lambda_{B}}, D^{\mu_{A}}\right)$, если существует $\lambda$-хорошая клетка $B$ вычета $\operatorname{res} A, u \operatorname{dim} \operatorname{Ext}_{\Sigma_{r}}^{1}\left(D^{\lambda}, D^{\mu}\right) \leqslant \varepsilon\left(\lambda, \mu_{A}\right)$ иначе.

ДокАЗАТЕЛЬСТво. Поскольку $\operatorname{Ext}_{\Sigma_{r-1}}^{1}\left(D^{\lambda}, D^{\lambda}\right)=0$, то достаточно считать, что $\lambda \neq \mu$. В силу результатов работ [14] и [15] имеем $D^{\lambda} \downarrow_{\Sigma_{r-1}} \cong \bigoplus\left\{D^{\lambda_{B}}: B-\right.$ $\lambda$-хорошая клетка $\}$. Поэтому в силу следствия 3 достаточно доказать, что из сушествования $\lambda$-хорошей клетки $B$ вычета res $A$ следует $\varepsilon\left(\lambda, \mu_{A}\right)=0$. Действительно, предположим противное. Тогда $\lambda=\left(\mu_{A}\right)^{C}$, где $\operatorname{res} A=\operatorname{res} C$ и $A$ выше $C$. Последнее возможно только тогда, когда $\chi(\lambda)=p, A=F+(0,1)$ и $C=L$, где $F$ и $L$ связаны с $\lambda$, как в лемме 8 . Так как в этом случае клетка $L$ не $\lambda$-хорошая, то не сушествует $\lambda$-хорошей клетки $B$ вычета res $A$. Противоречие.

Следующее утверждение объясняет, почему мы строили множество $X$ именно таким образом.

Лемма 10. Пусть $p>2 u \lambda, \mu-$ разбиения числа $r$ высоты менее $p$ такие, что $\lambda$ вполне расщепляемое, $\lambda \ngtr \mu u(\lambda, \mu) \notin X$. Тогда $\operatorname{Ext}_{\Sigma_{r}}^{1}\left(D^{\lambda}, D^{\mu}\right)=0$.

ДоКАЗАТЕЛЬСТВо. Проведем индукцию по $r$. Для $r=1$ это очевидно. Предположим, что лемма верна для разбиений чисел, меньших, чем $r>1$. Пусть $\lambda, \mu \vdash r$ такие, что $\lambda$ вполне расщепляемое, $\lambda \not \mu$ и $(\lambda, \mu) \notin X$. В силу утверждения 1 понятно, что при нарушении условия 1$)$ для пары $(\lambda, \mu)$ лемма доказана. Поэтому будем предполагать, что условие 1) выполнено, а условие 2) нарушено. Тогда сушествует некоторая $\mu$-хорошая клетка $A$ такая, что $\varepsilon\left(\lambda, \mu_{A}\right)=0$ и для любой $\lambda$-хорошей клетки $B$ выполнено $\left(\lambda_{B}, \mu_{A}\right) \notin X_{r-1}$.

Предположим, что сушествует некоторая $\lambda$-хорошая клетка $B$ вычета res $A$ такая, что $\operatorname{Ext}_{\Sigma_{r-1}}^{1}\left(D^{\lambda_{B}}, D^{\mu_{A}}\right) \neq 0$. В силу индуктивного предположения получаем $\lambda_{B} \triangleright \mu_{A}$. Докажем, что $\mu_{A}$ - вполне расщепляемое разбиение. В случае 
$h\left(\mu_{A}\right) \leqslant h\left(\lambda_{B}\right)$ это следует из того, что $\mu_{A} \triangleleft \lambda_{B}$. Поэтому можно считать, что $h\left(\mu_{A}\right)>h\left(\lambda_{B}\right)$. Однако из $\lambda \triangleleft \mu$ следует, что $h(\lambda) \geqslant h(\mu)$. Поэтому $h\left(\lambda_{B}\right)=$ $h(\lambda)-1$ и $\lambda_{1}+h(\lambda) \leqslant p$. Теперь то, что $\mu_{A}$ вполне расщепляемо, следует из неравенств $h\left(\mu_{A}\right) \leqslant h(\mu) \leqslant h(\lambda)$.

Поскольку

$$
\operatorname{Ext}_{\Sigma_{r-1}}^{1}\left(D^{\lambda_{B}}, D^{\mu_{A}}\right)=\operatorname{Ext}_{\Sigma_{r-1}}^{1}\left(D^{\mu_{A}}, D^{\lambda_{B}}\right) \neq 0
$$

то в силу индуктивного предположения получаем, что $\left(\mu_{A}, \lambda_{B}\right) \in X_{r-1}$. Из леммы 9 следует, что $\mu_{A}$ - большое разбиение и $\widetilde{\mu_{A}}=\lambda_{B}$. Так как оба разбиения $\mu_{A}$ и $\lambda_{B}$ вполне расшепляемые, то в силу определения 4 имеем $h\left(\lambda_{B}\right)=h\left(\mu_{A}\right)-1$. Тогда получаем

$$
h(\lambda) \geqslant h(\mu) \geqslant h\left(\mu_{A}\right)>h\left(\lambda_{B}\right) .
$$

Следовательно, $h(\lambda)=h\left(\lambda_{B}\right)+1$ и $h(\lambda)=h\left(\mu_{A}\right)$. Отсюда имеем $h(\lambda) \geqslant 2$ и $B=(h(\lambda), 1)$.

Обозначим через $x$ количество клеток, перемешаемых при переходе $\mu_{A} \mapsto \widetilde{\mu_{A}}=$ $\lambda_{B}$. Тогда имеем $\lambda_{1}=\left(\lambda_{B}\right)_{1} \geqslant\left(\mu_{A}\right)_{1}+x$. Понятно, что если $A$ не в первой строке или $x>1$, то $\lambda_{1}>\mu_{1}$. Это противоречит тому, что $\lambda \triangleleft \mu$. Предположим теперь, что $A$ в первой строке и $x=1$. Из последнего условия следует, что $\chi\left(\mu_{A}\right)=p$, и по определению 4 получаем $\lambda_{B}=\widetilde{\mu_{A}}=\left(\left(\mu_{A}\right)_{B}\right)^{A}=\mu_{B}$ и, следовательно, $\lambda=\mu$, что не так. Таким образом, и этот случай невозможен, и мы приходим к противоречию. В силу следствия 5 имеем $\operatorname{Ext}_{\Sigma_{r}}^{1}\left(D^{\lambda}, D^{\mu}\right)=0$.

Теорема 6. Пусть $p>2$ и $\lambda, \mu$-р-регулярные разбиения числа $r$ такие, что модуль $D^{\lambda}$ вполне расщепляемый $и \lambda \not \mu$. Тогда $\operatorname{Ext}_{\Sigma_{r}}^{1}\left(D^{\lambda}, D^{\mu}\right)$ одномерно, если $\lambda$-большое разбиение $и \mu=\tilde{\lambda}$, и равно нулю иначе.

ДокАЗАТЕЛЬСТво. Предположим сначала, что $h(\mu)<p$. Тогда в силу леммы 10 достаточно доказать, что $\operatorname{Ext}_{\Sigma_{r}}^{1}\left(D^{\lambda}, D^{\tilde{\lambda}}\right) \cong K$ для любого большого разбиения $\lambda$. Итак, пусть $\lambda$ - большое разбиение и $(h, j)$ - клетка последней строки диаграммы $[\lambda]$, как в определении 4 . Тогда мы имеем $h_{1, j}(\lambda)=p$ и $h_{2, j}(\lambda)<p$. Следовательно, в силу так называемой гипотезы Картера, доказанной в [9], модуль $S^{\lambda}$ не прост. Отсюда по леммам 9 и 10 единственным верхним композиционным фактором ненулевого модуля $\operatorname{rad} S^{\lambda}$ является $D^{\tilde{\lambda}}$. Следовательно, $\operatorname{dim} \operatorname{Ext}_{\Sigma_{r}}^{1}\left(D^{\lambda}, D^{\tilde{\lambda}}\right) \geqslant 1$. Обратное неравенство получается из следствия 5 .

Наконец, рассмотрим случай $h(\mu) \geqslant p$. Для этого заметим, что в доказательствах теорем 2.9 и 2.10 работы [13] ограничение $h(\mu)<p$ несущественно. Надо лишь установить, что $\mu=\varepsilon_{n}$ или $\mu=\kappa_{n}$ (после того, как предположено $\lambda_{A}=\varepsilon_{n-1}$ и $\gamma=\varepsilon_{n-1}$ ) без использования леммы 1.6 (той же работы).

Действительно, поскольку $\mu_{C} \unlhd \gamma=\varepsilon_{n-1}=\lambda_{A}$ и $\mu_{C} \Varangle \lambda_{A}$, то мы имеем $\mu_{C}=\varepsilon_{n-1}$. Это значит, что $\mu=\kappa_{n}$ или $\mu=\varepsilon_{n}$ или $\mu=\varepsilon_{n-1}^{D}$, где $D=(p, 1)$. Однако последний случай невозможен, так как $\varepsilon_{n-1}^{D} \triangleleft \varepsilon_{n}, \kappa_{n}$ и $\lambda=\varepsilon_{n}$ или $\lambda=\kappa_{n}$.

Доказанная теорема позволяет более точно контролировать процесс поднятия радикала, описанный в [18], чем это сделано в [3]. Докажем также следуюший результат. 
СлЕДСТВИЕ 6. Пусть $\lambda$ - вполне расщепляемое разбиение. Если $\lambda-$ большое разбиение, то $\operatorname{rad} S^{\lambda}$ является гомоморфным образом модуля $S^{\tilde{\lambda}}$ и при этом $\operatorname{rad} S^{\lambda}=\varphi\left(S^{\tilde{\lambda}}\right)$ для любого ненулевого гомоморфизма $\varphi: S^{\tilde{\lambda}} \rightarrow S^{\lambda}$. В противном случае $\operatorname{rad} S^{\lambda}=0$.

ДоКАЗАТЕЛЬСТво. В случае $h(\lambda)=1$ следствие, очевидно, выполнено. Поэтому мы будем считать, что $p>2$ и $h(\lambda)>1$. Если $\lambda$ - не большое разбиение, то в силу гипотезы Картера [9] модуль $S^{\lambda}$ прост и $\operatorname{rad} S^{\lambda}=0$.

Предположим теперь, что $\lambda$ - большое разбиение. В силу $[1, \S 24.10]$ существует некоторьй ненулевой гомоморфизм $\varphi: S^{\tilde{\lambda}} \rightarrow S^{\lambda}$. Положим $\mu=\lambda-\left(x^{h(\lambda)}\right)$, где $x=\lambda_{1}+h(\lambda)-p-1$. Легко видеть, что $h_{1,1}(\mu)=p, \mu \neq(p),\left(1^{p}\right)$ и $\tilde{\mu}+\left(x^{h(\lambda)}\right)=\tilde{\lambda}$. В силу леммы 11 и $[10$, теорема 6$]$ имеем

$$
1=\left[S^{\mu}: D^{m\left(\mu^{t}\right)}\right]=\left[S^{\mu}: D^{\tilde{\mu}}\right]=\left[S^{\lambda}: D^{\tilde{\mu}+\left(x^{h(\lambda)}\right)}\right]=\left[S^{\lambda}: D^{\tilde{\lambda}}\right] .
$$

Отсюда по теореме 6 получаем $\varphi\left(S^{\tilde{\lambda}}\right)=\operatorname{rad} S^{\lambda}$.

Обозначим через $m$ биекцию множества $p$-регулярных разбиений, определенную формулой $D^{\lambda} \otimes \operatorname{sgn}_{r} \cong D^{m(\lambda)}$. Комбинаторное описание отображения $m$ было предложено в [17] и доказано в [6].

ЛЕмма 11. Пусть $\lambda$ - разбиение, отличное от $(p)$ u $\left(1^{p}\right)$, такое, что $h_{1,1}(\lambda)=p$. Тогда $m\left(\lambda^{t}\right)=\tilde{\lambda}$.

ДоКАЗАТЕЛЬСТВо. Следуя работе [17], обозначим для любого разбиения $\mu$ через $I(\mu)$ разбиение, полученное из $\mu$ удалением его $p$-грани. В силу $[17,5.2]$ имеем $m\left(I\left(\lambda^{t}\right)\right)=\left(I\left(\lambda^{t}\right)\right)^{t}=I(\lambda)$. Теперь требуемая формула следует из $[17,4.1]$ и того, что $I(\tilde{\lambda})=I(\lambda)$ и $h(\tilde{\lambda})=h(\lambda)-1=p-\lambda_{1}$.

ЗАмЕчАниЕ 4 . В случае, когда $\lambda$ - большое разбиение, явная конструкция расширения $\operatorname{Ext}_{\Sigma_{r}}^{1}\left(D^{\lambda}, D^{\tilde{\lambda}}\right)$ реализуется в виде модуля $S^{\lambda} / \operatorname{rad} \operatorname{rad} S^{\lambda}$.

\section{Список литературы}

1. Джеймс Г. Теория представлений симметрической группы. М.: Мир, 1980.

2. Ламбек И. Кольца и модули. М.: Мир, 1971.

3. Щиголев В. В. Конечная базируемость некоторых классов неприводимых представлений симметрических групп // Матем. сб. 2003. Т. 194. №3. С. 149-160.

4. Brundan J., Kleshchev A.S. On translation functors for general linear and symmetric groups // Proc. London Math. Soc. (3). 2000. V. 80. № 1. P. 75-106.

5. Donkin S. On Schur algebras and related algebras. I // J. Algebra. 1986. V. 104. № 2. P. $310-328$.

6. Ford B., Kleshchev A.S. A proof of the Mullineux conjecture // Math. Z. 1997. V. 226. № 2. P. 267-308.

7. Green J. A. Polynomial representations of $\mathrm{GL}_{n}(K)$ // Lecture Notes in Math. V.830. Berlin-Heidelberg-N.Y.: Springer-Verlag, 1980.

8. Hemmer D. J. The Ext ${ }^{1}$-quiver for completely splittable representations of the symmetric group // J. Group Theory. 2001. № 4. P. 401-416.

9. James G.D., Murphy G.E. The determinant of the Gram matrix for a Specht module // J. Algebra. 1979. V. 59. № 1. P. 222-235.

10. James G.D. On the decomposition matrices of the symmetric groups. III // J. Algebra. 1981. V. 71. № 1. P. 115-122. 
11. Jantzen J. C. Representations of algebraic groups // Pure and Applied Mathematics. V.131. Boston: Academic Press, Inc., 1987.

12. Kleshchev A.S., Nakano D. On comparing the cohomology of general linear and symmetric groups // Pacific J. of Math. 2001. V. 201. P. 339-355.

13. Kleshchev A.S., Sheth J. On extensions of simple modules over symmetric and algebraic groups // J. Algebra. 1999. V. 221. № 2. P. 705-722.

14. Kleshchev A.S. Branching rules for modular representations of symmetric groups. I // J. Algebra. 1995. V. 178. № 2. P. 493-511.

15. Kleshchev A.S. Branching rules for modular representations of symmetric groups. II // J. Reine Angew. Math. 1995. V. 459. P. 163-212.

16. Kleshchev A.S. Completely splittable representations of symmetric groups // J. Algebra. 1996. V. 181. № 2. P. 584-592.

17. Mullineux $G$. Bijections of $p$-regular partitions and $p$-modular irreducibles of the symmetric groups // J. London Math. Soc. (2). 1979. V. 20. № 1. P. 60-66.

18. Shchigolev $V$. V. On the stabilization problem for submodules of Specht modules // J. Algebra. 2002. V. 251. № 2. P. 790-812.

Ульяновский государственный университет

Поступило в редакцию

E-mail: vkshch@vens.ru

07.05.2003 